\title{
Colloquium: Non-Markovian dynamics in open quantum systems
}

\author{
Heinz-Peter Breuer \\ Physikalisches Institut, Universität Freiburg, \\ Hermann-Herder-Straße 3, D-79104 Freiburg, Germany \\ Elsi-Mari Laine \\ QCD Labs, COMP Centre of Excellence, \\ Department of Applied Physics, Aalto University, \\ P.O. Box 13500, FI-00076 AALTO, Finland \\ and Turku Centre for Quantum Physics, \\ Department of Physics and Astronomy, \\ University of Turku, Fl-20014 Turun yliopisto, Finland
}

Jyrki Piilo

Turku Centre for Quantum Physics, Department of Physics and Astronomy, University of Turku, Fl-20014 Turun yliopisto, Finland

\author{
Bassano Vacchini \\ Dipartimento di Fisica, Università degli Studi di Milano, \\ Via Celoria 16, I-20133 Milan, Italy \\ and INFN, Sezione di Milano, Via Celoria 16, I-20133 Milan, Italy
}

(published 19 April 2016)

\begin{abstract}
The dynamical behavior of open quantum systems plays a key role in many applications of quantum mechanics, examples ranging from fundamental problems, such as the environment-induced decay of quantum coherence and relaxation in many-body systems, to applications in condensed matter theory, quantum transport, quantum chemistry, and quantum information. In close analogy to a classical Markovian stochastic process, the interaction of an open quantum system with a noisy environment is often modeled phenomenologically by means of a dynamical semigroup with a corresponding timeindependent generator in Lindblad form, which describes a memoryless dynamics of the open system typically leading to an irreversible loss of characteristic quantum features. However, in many applications open systems exhibit pronounced memory effects and a revival of genuine quantum properties such as quantum coherence, correlations, and entanglement. Here recent theoretical results on the rich non-Markovian quantum dynamics of open systems are discussed, paying particular attention to the rigorous mathematical definition, to the physical interpretation and classification, as well as to the quantification of quantum memory effects. The general theory is illustrated by a series of physical examples. The analysis reveals that memory effects of the open system dynamics reflect characteristic features of the environment which opens a new perspective for applications, namely, to exploit a small open system as a quantum probe signifying nontrivial features of the environment it is interacting with. This Colloquium further explores the various physical sources of non-Markovian quantum dynamics, such as structured environmental spectral densities, nonlocal correlations between environmental degrees of freedom, and correlations in the initial system-environment state, in addition to developing schemes for their local detection. Recent experiments addressing the detection, quantification, and control of non-Markovian quantum dynamics are also briefly discussed.
\end{abstract}

DOI: 10.1103/RevModPhys.88.021002

\section{CONTENTS}

I. Introduction 2

II. Definitions and Measures for Quantum Non-Markovianity

A. Basic concepts

1. Open quantum systems and dynamical maps

2. Divisibility and time-local master equations
B. Classical versus quantum non-Markovianity

1. Classical stochastic processes and the Markov condition

2. Non-Markovianity in the quantum regime

C. Quantum non-Markovianity and information flow

1. Trace distance and distinguishability of quantum states

2. Definition and quantification of memory effects

3. Generalizing the trace distance measure

*breuer@physik.uni-freiburg.de 
4. Connection between quantum and classical non-Markovianity

D. Alternative approaches

1. Quantum non-Markovianity and $C P$ divisibility

2. Monotonicity of correlations and entropic quantities

III. Models and Applications of Non-Markovianity

A. Prototypical model systems

1. Pure decoherence model

2. Two-level system in a dissipative environment

3. Single qubit with multiple decoherence channels

4. Spin-boson model

B. Applications of quantum non-Markovianity

1. Analysis and control of non-Markovian dynamics

2. Open systems as non-Markovian quantum probes

IV. Impact of Correlations and Experimental Realizations

A. Initial system-environment correlations

1. Initial correlations and dynamical maps

2. Local detection of initial correlations

B. Nonlocal memory effects

C. Experiments on non-Markovianity and correlations

1. Control and quantification of memory effects in photonic systems

2. Experiments on the local detection of correlations

3. Non-Markovian quantum probes detecting nonlocal correlations in composite environments

V. Summary and Outlook

Acknowledgments

References

\section{INTRODUCTION}

The observation and experimental control of characteristic quantum properties of physical systems is often strongly hindered by the coupling of the system to a noisy environment. The unavoidable interaction of the quantum system with its surroundings generates system-environment correlations leading to an irretrievable loss of quantum coherence. Realistic quantum mechanical systems are thus open systems governed by a nonunitary time development which describes all features of irreversible dynamics such as the dissipation of energy, the relaxation to a thermal equilibrium or a stationary nonequilibrium state, and the decay of quantum coherences and correlations (Davies, 1976; Alicki and Lendi, 1987; Breuer and Petruccione, 2002).

There is a well-established treatment of the dynamics of open quantum systems in which the open system's time evolution is described by a master equation, a first-order differential equation for the reduced density matrix of the open system. In the simplest case the generator of this master equation is time independent which leads to a dynamical semigroup representing the dynamics of the open system. According to general mathematical and physical principles such master equations must have a very specific structure known as Lindblad structure (Gorini, Kossakowski, and Sudarshan, 1976; Lindblad, 1976). If one adopts a microscopic system-environment approach to the dynamics of open systems, master equations of this structure may be derived, for example, with the help of the Born-Markov approximation which is based on second-order perturbation theory with respect to the system-environment coupling and further assumes short environmental correlation times. However, it turns out that for many processes in open quantum systems the approximations underlying this approach are not satisfied and that a description of the dynamics by means of a dynamical semigroup fails. Typically, this is due to the fact that the relevant environmental correlation times are not small compared to the system's relaxation or decoherence time, rendering the standard Markov approximation impossible. The violation of this separation of time scales can occur, for example, in the cases of strong system-environment couplings, structured or finite reservoirs, low temperatures, or large initial systemenvironment correlations.

If the dynamics of an open quantum system substantially deviates from that of a dynamical semigroup, one often speaks of a non-Markovian process. This term refers to a well-known concept of the theory of classical stochastic processes and is used to loosely indicate the presence of memory effects in the time evolution of the open system. However, the classical notions of Markovianity and nonMarkovianity cannot be transferred to the quantum regime in a natural way since they are based on concepts of classical probability theory which are not applicable in quantum mechanics (Lindblad, 1979; Accardi, Frigerio, and Lewis, 1982; Vacchini et al., 2011). Therefore, the concept of a quantum non-Markovian process requires a precise definition which cannot be based on classical notions only. Many important questions need to be discussed in this context: How can one rigorously define non-Markovian dynamics in the quantum case, how do quantum memory effects manifest themselves in the behavior of open systems, and can such effects be uniquely identified experimentally through monitoring of the open system? The definition of non-Markovianity should provide a general mathematical characterization which does not rely on any specific representation or approximation of the dynamics, e.g., in terms of a perturbative master equation. Moreover, definitions of non-Markovianity should lead to quantitative measures for the degree of non-Markovianity, enabling the comparison of the amount of memory effects in different physical systems.

Recently, a series of different proposals to answer these questions was published, rigorously defining the border between the regions of Markovian and non-Markovian quantum dynamics and developing quantitative measures for the degree of memory effects [see, e.g., Wolf et al. (2008), Breuer, Laine, and Piilo (2009), Rivas, Huelga, and Plenio (2010), Chruściński, Kossakowski, and Rivas (2011), Luo, Fu, and Song (2012), and Chruściński and Maniscalco (2014), the tutorial paper by Breuer (2012), and the recent review by Rivas, Huelga, and Plenio (2014)]. Here we describe and discuss several of these ideas, paying particular attention to those concepts which are based on the exchange of information between the open system and its environment, and on the divisibility of the dynamical map describing the open system's time evolution. We also explain the relations between the 
classical and the quantum notions of non-Markovianity and develop a general classification of quantum dynamical maps which is based on these concepts.

The general theory will be illustrated by a series of examples. We start with simple prototypical models describing pure decoherence dynamics, dissipative processes, relaxation through multiple decay channels, and the spin-boson problem. We then continue with the study of the dynamics of open systems which are coupled to interacting many-body environments. The examples include an Ising and a Heisenberg chain in transverse magnetic fields, as well as an impurity atom in a Bose-Einstein condensate (Apollaro et al., 2011; Haikka et al., 2011, 2012). The discussion will demonstrate, in particular, that memory effects of the open system dynamics reflect characteristic properties of the environment. This fact opens a new perspective, namely, to exploit a small open system as a quantum probe signifying nontrivial features of a complex environment, for example, the critical point of a phase transition (Smirne, Cialdi et al., 2013; Gessner, Ramm, Häffner et al., 2014). Another example to be discussed here is the use of nonMarkovian dynamics to determine nonlocal correlations within a composite environment carrying out only measurements on the open system functioning as a quantum probe (Laine et al., 2012; Liu et al., 2013; Wißmann and Breuer, 2014).

A large variety of further applications of quantum memory effects is described in the literature. Interested readers can find examples dealing with, e.g., phenomenological master equations (Mazzola et al., 2010), quantum Brownian motion in optomechanical systems (Gröblacher et al., 2015), chaotic systems (Znidaric, Pineda, and García-Mata, 2011), energy transfer processes in photosynthetic complexes (Rebentrost and Aspuru-Guzik, 2011), continous variable quantum key distribution (Vasile, Olivares et al., 2011), metrology (Chin, Huelga, and Plenio, 2012), steady state entanglement (Huelga, Rivas, and Plenio, 2012), Coulomb crystals (Borrelli et al., 2013), symmetry breaking (Chancellor, Petri, and Haas, 2013), and time-invariant quantum discord (Haikka, Johnson, and Maniscalco, 2013).

The standard description of the open system dynamics in terms of a dynamical map is based on the assumption of an initially factorizing system-environment state. The approach developed here also allows one to investigate the impact of correlations in the initial system-environment state and leads to schemes for the local detection of such correlations through monitoring of the open system (Laine, Piilo, and Breuer, 2010b; Gessner and Breuer, 2011).

In recent years, several of the above features of nonMarkovian quantum dynamics have been observed experimentally in photonic and trapped ion systems (Li et al., 2011; Liu et al., 2011; Smirne et al., 2011; Cialdi et al., 2014; Gessner, Ramm, Pruttivarasin et al., 2014; Tang et al., 2015). We briefly discuss the results of these experiments which demonstrate the transition from Markovian to non-Markovian quantum dynamics, the non-Markovian behavior induced by nonlocal environmental correlations, and the local scheme for the detection of nonclassical initial system-environment correlations.

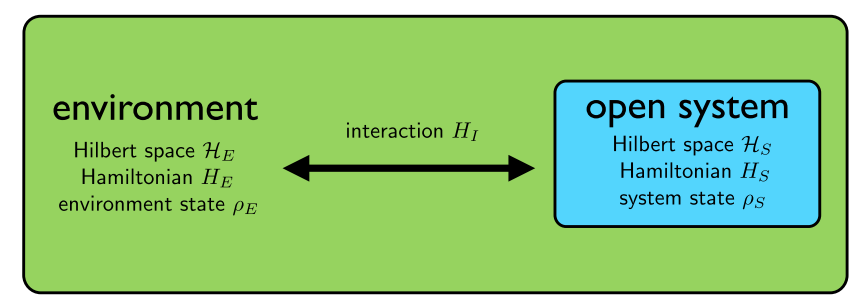

FIG. 1. An open quantum system described by the Hilbert space $\mathcal{H}_{S}$ and the Hamiltonian $H_{S}$, which is coupled to an environment with Hilbert space $\mathcal{H}_{E}$ and Hamiltonian $H_{E}$ through an interaction Hamiltonian $H_{I}$.

\section{DEFINITIONS AND MEASURES FOR QUANTUM NON-MARKOVIANITY}

\section{A. Basic concepts}

\section{Open quantum systems and dynamical maps}

An open quantum system $S$ (Davies, 1976; Alicki and Lendi, 1987; Breuer and Petruccione, 2002) can be regarded as a subsystem of some larger system composed of $S$ and another subsystem $E$, its environment; see Fig. 1. The Hilbert space of the total system $S+E$ is given by the tensor product space

$$
\mathcal{H}_{S E}=\mathcal{H}_{S} \otimes \mathcal{H}_{E}
$$

where $\mathcal{H}_{S}$ and $\mathcal{H}_{E}$ denote the Hilbert spaces of $S$ and $E$, respectively. Physical states of the total system are represented by positive trace class operators $\rho_{S E}$ on $\mathcal{H}_{S E}$ with unit trace, satisfying $\rho_{S E} \geq 0$ and $\operatorname{tr} \rho_{S E}=1$. We recall that a Hermitian operator $A$ is said to be positive, $A \geq 0$, if all of its eigenvalues are positive. Given a state of the total system, the corresponding states of subsystems $S$ and $E$ are obtained by partial traces over $\mathcal{H}_{E}$ and $\mathcal{H}_{S}$, respectively, i.e., we have $\rho_{S}=\operatorname{tr}_{E} \rho_{S E}$ and $\rho_{E}=\operatorname{tr}_{S} \rho_{S E}$. We denote the convex set of physical states belonging to some Hilbert space $\mathcal{H}$ by $\mathcal{S}(\mathcal{H})$.

We suppose that the total system $S+E$ is closed and governed by a Hamiltonian of the general form

$$
H=H_{S} \otimes I_{E}+I_{S} \otimes H_{E}+H_{I}
$$

where $H_{S}$ and $H_{E}$ are the free Hamiltonians of system and environment, respectively, and $H_{I}$ is an interaction Hamiltonian. The corresponding unitary time evolution operator is thus given by

$$
U(t)=\exp (-i H t) \quad(\hbar=1)
$$

The dynamics of the total system states is obtained from the von Neumann equation

$$
\frac{d}{d t} \rho_{S E}(t)=-i\left[H, \rho_{S E}(t)\right]
$$

which yields the formal solution

$$
\rho_{S E}(t)=\mathcal{U}_{t} \rho_{S E}(0) \equiv U(t) \rho_{S E}(0) U^{\dagger}(t)
$$


To simplify the presentation we assumed time-independent Hamiltonians in Eq. (2). We emphasize however, that all concepts discussed in the following are also valid for timedependent Hamiltonians. In particular, the system Hamiltonian $H_{S}=H_{S}(t)$ may depend explicitly on time involving, for example, an arbitrary external control field.

An important concept in the theory of open quantum systems is that of a dynamical map. To explain this concept we assume that the initial state of the total system is an uncorrelated tensor product state

$$
\rho_{S E}(0)=\rho_{S}(0) \otimes \rho_{E}(0)
$$

which leads to the following expression for the reduced open system state at any time $t \geq 0$ :

$$
\rho_{S}(t)=\operatorname{tr}_{E}\left\{U(t) \rho_{S}(0) \otimes \rho_{E}(0) U^{\dagger}(t)\right\}
$$

Considering a fixed initial environmental state $\rho_{E}(0)$ and any fixed time $t \geq 0$, Eq. (7) defines a linear map

$$
\Phi_{t}: S\left(\mathcal{H}_{S}\right) \rightarrow S\left(\mathcal{H}_{S}\right)
$$

on the open system's state space $S\left(\mathcal{H}_{S}\right)$ which maps any initial open system state $\rho_{S}(0)$ to the corresponding open system state $\rho_{S}(t)$ at time $t$ :

$$
\rho_{S}(0) \mapsto \rho_{S}(t)=\Phi_{t} \rho_{S}(0)
$$

$\Phi_{t}$ is called a quantum dynamical map. It is easy to verify that it preserves the Hermiticity and the trace of operators, and that it is a positive map, i.e., that it maps positive operators to positive operators. Thus, $\Phi_{t}$ maps physical states to physical states.

A further important property of the dynamical map $\Phi_{t}$ is that it is not only positive but also completely positive. Maps with this property are also known as trace preserving quantum operations or quantum channels in quantum information theory (Nielsen and Chuang, 2000). Completely positive maps play an important role, for example, in the development of fault-tolerant quantum error correction codes [see, e.g., Alicki, Lidar, and Zanardi (2006)]. Let us recall that a linear map $\Phi$ is completely positive if and only if it admits a Kraus representation (Kraus, 1983), which means that there are operators $\Omega_{i}$ on the underlying Hilbert space $\mathcal{H}_{S}$ such that $\Phi A=\sum_{i} \Omega_{i} A \Omega_{i}^{\dagger}$, and that the condition of trace preservation takes the form $\sum_{i} \Omega_{i}^{\dagger} \Omega_{i}=I_{S}$. An equivalent definition of complete positivity is the following (Nielsen and Chuang, 2000). We consider for any number $n=1,2, \ldots$ the tensor product space $\mathcal{H}_{S} \otimes \mathbb{C}^{n}$ which represents the Hilbert space of $S$ combined with an $n$-level system $R$. We can define a map $\Phi \otimes I_{n}$ operating on the combined system by a linear extension of the relation $\left(\Phi \otimes I_{n}\right)(A \otimes B)=(\Phi A) \otimes B$. This map $\Phi \otimes I_{n}$ thus describes a quantum operation of the composite system which acts nontrivially only on the first factor representing subsystem $S$. One then defines the map $\Phi$ to be $n$ positive if $\Phi \otimes I_{n}$ is a positive map, and completely positive if $\Phi \otimes I_{n}$ is a positive map for all $n$. We note that positivity is equivalent to 1-positivity, and that for a Hilbert space with finite dimension $N_{S}=\operatorname{dim} \mathcal{H}_{S}$ complete positivity is equivalent to $N_{S}$-positivity.

Thus, complete positivity is a stronger condition than positivity. Positivity of a map $\Phi$ guarantees that physical states $\rho_{S}$ are mapped to physical states $\Phi \rho_{S}$, i.e., positivity means that probabilities stay positive under the action of the map. However, if $\rho_{S}$ describes the state of some subsystem $S$ of a larger system $S+R$, it can happen that physical states of $S+R$ are transformed under $\Phi$ into Hermitian operators which do not represent physical states since they involve negative probabilities. This is a genuine quantum feature, which does not occur in classical physics and which is intimately connected with the presence of entanglement between $S$ and $R$. The stronger property of complete positivity of $\Phi$ ensures not only that all physical states of $S$ are mapped to physical states of $S$, but also that all physical states of $S+R$ are mapped to physical states of $S+R$.

If the time parameter $t$ now varies over some time interval from 0 to $T$, where $T$ may be finite or infinite, we obtain a one-parameter family of dynamical maps,

$$
\Phi=\left\{\Phi_{t} \mid 0 \leq t \leq T, \Phi_{0}=I\right\}
$$

where $I$ denotes the unit map, and the initial environmental state $\rho_{E}(0)$ used to construct $\Phi_{t}$ is still kept fixed. This family contains the complete information on the dynamics of all initial open system states over the time interval $[0, T]$ we are interested in.

\section{Divisibility and time-local master equations}

Let us suppose that the inverse of $\Phi_{t}$ exists for all times $t \geq 0$. We can then define a two-parameter family of maps by means of

$$
\Phi_{t, s}=\Phi_{t} \Phi_{s}^{-1}, \quad t \geq s \geq 0
$$

such that we have $\Phi_{t, 0}=\Phi_{t}$ and

$$
\Phi_{t, 0}=\Phi_{t, s} \Phi_{s, 0}
$$

The existence of the inverse for all positive times thus allows us to introduce the very notion of divisibility. While $\Phi_{t, 0}$ and $\Phi_{s, 0}$ are completely positive by construction, the map $\Phi_{t, s}$ need not be completely positive and not even positive since the inverse $\Phi_{s}^{-1}$ of a completely positive map $\Phi_{s}$ need not be positive. The family of dynamical maps is said to be $P$ divisible if $\Phi_{t, s}$ is positive, and $C P$ divisible if $\Phi_{t, s}$ is completely positive for all $t \geq s \geq 0$. A simple example for a $C P$-divisible process is provided by a semigroup $\Phi_{t}=e^{\mathcal{L} t}$ with a generator $\mathcal{L}$ in Lindblad form. In this case we have $\Phi_{t, s}=e^{\mathcal{L}(t-s)}$ which is trivially completely positive. As discussed later on there are many physically relevant models which give rise to dynamical maps which are neither $C P$ divisible nor $P$ divisible.

An interesting property of the class of processes for which $\Phi_{t}^{-1}$ exists is given by the fact that they always lead to a timelocal quantum master equation for the open system states with the general structure 


$$
\begin{aligned}
\frac{d}{d t} \rho_{S}= & \mathcal{K}_{t} \rho_{S} \\
= & -i\left[H_{S}(t), \rho_{S}\right]+\sum_{i} \gamma_{i}(t)\left[A_{i}(t) \rho_{S} A_{i}^{\dagger}(t)\right. \\
& \left.-\frac{1}{2}\left\{A_{i}^{\dagger}(t) A_{i}(t), \rho_{S}\right\}\right]
\end{aligned}
$$

Master equations of this form can be derived, e.g., by employing the time-convolutionless projection operator technique (Shibata, Takahashi, and Hashitsume, 1977; Chaturvedi and Shibata, 1979). The master equation (13) is very similar to a Lindblad master equation, where however the Hamiltonian contribution $H_{S}(t)$, the Lindblad operators $A_{i}(t)$ (which can be supposed to be linearly independent), and the decay rates $\gamma_{i}(t)$ may depend on time since the process does not represent a semigroup, in general. Note that Eq. (13) involves a timedependent generator $\mathcal{K}_{t}$, but no convolution of the open system states with a memory kernel as in the NakajimaZwanzig equation (Nakajima, 1958; Zwanzig, 1960).

It is an important open problem to formulate general necessary and sufficient conditions under which the master equation (13) leads to completely positive dynamics. If the process represents a semigroup, the Hamiltonian $H_{S}$, the operators $A_{i}$, and the rates $\gamma_{i}$ must be time independent and a necessary and sufficient condition for complete positivity of the dynamics is simply that all decay rates are positive $\gamma_{i} \geq 0$. This is the famous Gorini-Kossakowski-Sudarshan-Lindblad theorem (Gorini, Kossakowski, and Sudarshan, 1976; Lindblad, 1976). However, we will see later by means of several examples that in the time-dependent case the rates $\gamma_{i}(t)$ can indeed become temporarily negative without violating complete positivity.

On the other hand, for divisible quantum processes it is indeed possible to formulate necessary and sufficient conditions. In fact, the master equation (13) leads to a $C P$ divisible dynamics if and only if all rates are positive for all times, $\gamma_{i}(t) \geq 0$, which follows from a straightforward extension of the Gorini-Kossakowski-Sudarshan-Lindblad theorem. Moreover, the master equation (13) leads to a $P$-divisible dynamics if and only if the weaker conditions

$$
\sum_{i} \gamma_{i}(t)\left|\left\langle n\left|A_{i}(t)\right| m\right\rangle\right|^{2} \geq 0
$$

hold for all orthonormal bases $\{|n\rangle\}$ of the open system and all $n \neq m$. This statement can be obtained by applying a characterization of the generators of positive semigroups due to Kossakowski (1972a, 1972b).

\section{B. Classical versus quantum non-Markovianity}

\section{Classical stochastic processes and the Markov condition}

In classical probability theory (Gardiner, 1985; van Kampen, 1992) a stochastic process $X(t), t \geq 0$, taking values in a discrete set $\left\{x_{i}\right\}_{i \in \mathbb{N}}$ is characterized by a hierarchy of joint probability distributions $P_{n}=P_{n}\left(x_{n}, t_{n} ; x_{n-1}, t_{n-1} ; \ldots ; x_{1}, t_{1}\right)$ for all $n \in \mathbb{N}$ and times $t_{n} \geq t_{n-1} \geq \cdots \geq t_{1} \geq 0$, known as Kolmogorov hierarchy. The distribution $P_{n}$ yields the probability that the process takes on the value $x_{1}$ at time $t_{1}$, the value $x_{2}$ at time $t_{2}, \ldots$, and the value $x_{n}$ at time $t_{n}$. In order for such a hierarchy to represent a stochastic process the Kolmogorov consistency conditions must be satisfied which, apart from conditions of positivity and normalization, require, in particular,

$\sum_{x_{m}} P_{n}\left(x_{n}, t_{n} ; \ldots ; x_{m}, t_{m} ; \ldots ; x_{1}, t_{1}\right)=P_{n-1}\left(x_{n}, t_{n} ; \ldots ; x_{1}, t_{1}\right)$,

connecting the $n$-point probability distribution $P_{n}$ to the $(n-1)$-point probability distribution $P_{n-1}$.

A stochastic process $X(t)$ is said to be Markovian if the conditional probabilities defined by

$P_{1 \mid n}\left(x_{n+1}, t_{n+1} \mid x_{n}, t_{n} ; \ldots ; x_{1}, t_{1}\right)=\frac{P_{n+1}\left(x_{n+1}, t_{n+1} ; \ldots ; x_{1}, t_{1}\right)}{P_{n}\left(x_{n}, t_{n} ; \ldots ; x_{1}, t_{1}\right)}$

satisfy

$P_{1 \mid n}\left(x_{n+1}, t_{n+1} \mid x_{n}, t_{n} ; \ldots ; x_{1}, t_{1}\right)=P_{1 \mid 1}\left(x_{n+1}, t_{n+1} \mid x_{n}, t_{n}\right)$.

This is the classical Markov condition which means that the probability for the stochastic process to take the value $x_{n+1}$ at time $t_{n+1}$, under the condition that it assumed values $x_{i}$ at previous times $t_{i}$, depends only on the last previous value $x_{n}$ at time $t_{n}$. In this sense the process is said to have no memory, since the past history prior to $t_{n}$ is irrelevant to determine the future once we know the value $x_{n}$ the process assumed at time $t_{n}$. Note that Eq. (17) imposes an infinite number of conditions for all $n$-point probability distributions which cannot be checked if only a few low-order distributions are known.

The Markov condition (17) substantially simplifies the mathematical description of stochastic processes. In fact, one can show that under this condition the whole hierarchy of joint probability distributions can be reconstructed from the initial one-point distribution $P_{1}\left(x_{0}, 0\right)$ and the conditional transition probability

$$
T(x, t \mid y, s) \equiv P_{1 \mid 1}(x, t \mid y, s)
$$

by means of

$$
\begin{aligned}
P_{n}\left(x_{n}, t_{n} ; x_{n-1}, t_{n-1} ; \ldots ; x_{1}, t_{1}\right) \\
\quad=\prod_{i=1}^{n-1} T\left(x_{i+1}, t_{i+1} \mid x_{i}, t_{i}\right) P_{1}\left(x_{1}, t_{1}\right)
\end{aligned}
$$

and

$$
P_{1}\left(x_{1}, t_{1}\right)=\sum_{x_{0}} T\left(x_{1}, t_{1} \mid x_{0}, 0\right) P_{1}\left(x_{0}, 0\right) .
$$

For a Markov process the transition probability has to obey the Chapman-Kolmogorov equation 


$$
T(x, t \mid y, s)=\sum_{z} T(x, t \mid z, \tau) T(z, \tau \mid y, s)
$$

for $t \geq \tau \geq s$. Thus, a classical Markov process is uniquely characterized by a probability distribution for the initial states of the process and a conditional transition probability satisfying the Chapman-Kolmogorov equation (21). Indeed, the latter provides the necessary condition in order to ensure that the joint probabilities defined by Eq. (19) satisfy the condition (15), so that they actually define a classical Markov process. Provided the conditional transition probability is differentiable with respect to time (which will always be assumed here) one obtains an equivalent differential equation, namely, the Chapman-Kolmogorov equation:

$$
\frac{d}{d t} T(x, t \mid y, s)=\sum_{z}\left[W_{x z}(t) T(z, t \mid y, s)-W_{z x}(t) T(x, t \mid y, s)\right],
$$

where $W_{z x}(t) \geq 0$ represents the rate (probability per unit of time) for a transition to the state $z$ given that the state is $x$ at time $t$. An equation of the same structure holds for the onepoint probability distribution of the process:

$$
\frac{d}{d t} P_{1}(x, t)=\sum_{z}\left[W_{x z}(t) P_{1}(z, t)-W_{z x}(t) P_{1}(x, t)\right],
$$

which is known as a Pauli master equation for a classical Markov process. The conditional transition probability of the process can be obtained solving Eq. (23) with the initial condition $P_{1}(x, s)=\delta_{x y}$.

\section{Non-Markovianity in the quantum regime}

This definition of a classical Markov process cannot be transferred immediately to the quantum regime (Vacchini et al., 2011). In order to illustrate the arising difficulties let us consider an open quantum system as described in Sec. II.A. Suppose we carry out projective measurements of an open system observable $\hat{X}$ at times $t_{n} \geq t_{n-1} \geq \cdots \geq t_{1} \geq 0$. For simplicity we assume that this observable is nondegenerate with spectral decomposition $\hat{X}=\sum_{x} x\left|\varphi_{x}\right\rangle\left\langle\varphi_{x}\right|$. As in Eq. (5) we write the unitary evolution superoperator as $\mathcal{U}_{t} \rho_{S E}=$ $U_{t} \rho_{S E} U_{t}^{\dagger}$ and the quantum operation corresponding to the measurement outcome $x$ as $\mathcal{M}_{x} \rho_{S E}=\left|\varphi_{x}\right\rangle\left\langle\varphi_{x}\left|\rho_{S E}\right| \varphi_{x}\right\rangle\left\langle\varphi_{x}\right|$. Applying the Born rule and the projection postulate one can then write a joint probability distribution

$$
\begin{aligned}
& P_{n}\left(x_{n}, t_{n} ; x_{n-1}, t_{n-1} ; \ldots ; x_{1}, t_{1}\right) \\
& \quad=\operatorname{tr}\left\{\mathcal{M}_{x_{n}} \mathcal{U}_{t_{n}-t_{n-1}} \cdots \mathcal{M}_{x_{1}} \mathcal{U}_{t_{1}} \rho_{S E}(0)\right\}
\end{aligned}
$$

which yields the probability of observing a certain sequence $x_{n}, x_{n-1}, \ldots, x_{1}$ of measurement outcomes at the respective times $t_{n}, t_{n-1}, \ldots, t_{1}$. Thus, it is of course possible in quantum mechanics to define a joint probability distribution for any sequence of measurement results. However, as is well known these distribution do in general not satisfy Eq. (15) since measurements carried out at intermediate times in general destroy quantum interferences. The fact that the joint probability distributions (24) violate in general the Kolmogorov condition (15) is even true for a closed quantum system. Thus, the quantum joint probability distributions given by Eq. (24) do not represent a classical hierarchy of joint probabilities satisfying the Kolmogorov consistency conditions. More generally, one can consider other joint probability distributions corresponding to different quantum operations describing nonprojective, generalized measurements.

For an open system coupled to some environment measurements performed on the open system not only influence quantum interferences but also system-environment correlations. For example, if the system-environment state prior to a projective measurement at time $t_{i}$ is given by $\rho_{S E}\left(t_{i}\right)$, the state after the measurement conditioned on the outcome $x$ is given by

$$
\rho_{S E}^{\prime}\left(t_{i}\right)=\frac{\mathcal{M}_{x} \rho_{S E}\left(t_{i}\right)}{\operatorname{tr} \mathcal{M}_{x} \rho_{S E}\left(t_{i}\right)}=\left|\varphi_{x}\right\rangle\left\langle\varphi_{x}\right| \otimes \rho_{E}^{x}\left(t_{i}\right),
$$

where $\rho_{E}^{x}$ is an environmental state which may depend on the measurement result $x$. Hence, projective measurements completely destroy system-environment correlations, leading to an uncorrelated tensor product state of the total system, and, therefore, strongly influence the subsequent dynamics.

We conclude that an intrinsic characterization and quantification of memory effects in the dynamics of open quantum systems, which is independent of any prescribed measurement scheme influencing the time evolution, has to be based solely on the properties of the dynamics of the open system's density matrix $\rho_{S}(t)$.

\section{Quantum non-Markovianity and information flow}

The first approach to quantum non-Markovianity to be discussed here is based on the idea that memory effects in the dynamics of open systems are linked to the exchange of information between the open system and its environment: While in a Markovian process the open system continuously loses information to the environment, a non-Markovian process is characterized by a flow of information from the environment back into the open system (Breuer, Laine, and Piilo, 2009; Laine, Piilo, and Breuer, 2010a). In such a way quantum non-Markovianity is associated with a notion of quantum memory, namely, information which has been transferred to the environment, in the form of system-environment correlations or changes in the environmental states, and is later retrieved by the system. To make this idea more precise we employ an appropriate distance measure for quantum states.

\section{Trace distance and distinguishability of quantum states}

The trace norm of a trace class operator $A$ is defined by $\|A\|=\operatorname{tr}|A|$, where the modulus of the operator is given by $|A|=\sqrt{A^{\dagger} A}$. If $A$ is self-adjoint the trace norm can be expressed as the sum of the moduli of the eigenvalues $a_{i}$ of $A$ counting multiplicities: $\|A\|=\sum_{i}\left|a_{i}\right|$. This norm leads to a natural measure for the distance between two quantum states $\rho^{1}$ and $\rho^{2}$ known as trace distance (Heinosaari and Ziman, 2011):

$$
D\left(\rho^{1}, \rho^{2}\right)=\frac{1}{2}\left\|\rho^{1}-\rho^{2}\right\| .
$$


The trace distance represents a metric on the state space $\mathcal{S}(\mathcal{H})$ of the underlying Hilbert space $\mathcal{H}$. We have the bounds $0 \leq D\left(\rho^{1}, \rho^{2}\right) \leq 1$, where $D\left(\rho^{1}, \rho^{2}\right)=0$ if and only if $\rho^{1}=\rho^{2}$, and $D\left(\rho^{1}, \rho^{2}\right)=1$ if and only if $\rho^{1}$ and $\rho^{2}$ are orthogonal. Recall that two density matrices are said to be orthogonal if their supports, i.e., the subspaces spanned by their eigenstates with nonzero eigenvalue, are orthogonal. The trace distance has a series of interesting mathematical and physical features which make it a useful distance measure in quantum information theory (Nielsen and Chuang, 2000). There are two properties which are the most relevant for our purposes.

The first property is that the trace distance between two quantum states admits a clear physical interpretation in terms of the distinguishability of these states. To explain this interpretation consider two parties, Alice and Bob, and suppose that Alice prepares a quantum system in one of two states $\rho^{1}$ or $\rho^{2}$, with a probability of $1 / 2$ each, and sends the system to Bob. The task of Bob is to find out by means of a single quantum measurement whether the system is in the state $\rho^{1}$ or $\rho^{2}$. It can be shown that the maximal success probability Bob can achieve through an optimal strategy is directly connected to the trace distance (Fuchs and van de Graaf, 1999):

$$
P_{\max }=\frac{1}{2}\left[1+D\left(\rho^{1}, \rho^{2}\right)\right] .
$$

Thus, we see that the trace distance represents the bias in favor of a correct state discrimination by Bob. For this reason the trace distance $D\left(\rho^{1}, \rho^{2}\right)$ can be interpreted as the distinguishability of the quantum states $\rho^{1}$ and $\rho^{2}$. For example, suppose the states prepared by Alice are orthogonal such that we have $D\left(\rho^{1}, \rho^{2}\right)=1$. In this case we get $P_{\max }=1$, which is a wellknown fact since orthogonal states can be distinguished with certainty by a single measurement, an optimal strategy of Bob being to measure the projection onto the support of $\rho^{1}$ or $\rho^{2}$.

The second important property of the trace distance is given by the fact that any completely positive and trace preserving map $\Lambda$ is a contraction for the trace distance, i.e., we have (Ruskai, 1994)

$$
D\left(\Lambda \rho^{1}, \Lambda \rho^{2}\right) \leq D\left(\rho^{1}, \rho^{2}\right)
$$

for all states $\rho^{1,2}$. In view of the interpretation of the trace distance we thus conclude that a trace preserving quantum operation can never increase the distinguishability of any two quantum states. We remark that the equality sign in Eq. (28) holds if $\Lambda$ is a unitary transformation, and that Eq. (28) is also valid for trace preserving maps which are positive but not completely positive.

\section{Definition and quantification of memory effects}

In Sec. II.C.1 we interpreted the trace distance of two quantum states as the distinguishability of these states, where it was assumed that the quantum state Bob receives is identical to the state prepared by Alice. Suppose now that Alice prepares her states $\rho_{S}^{1,2}(0)$ as initial states of an open quantum system $S$ coupled to some environment $E$. Bob will then receive at time $t$ the system in one of the states $\rho_{S}^{1,2}(t)=\Phi_{t} \rho_{S}^{1,2}(0)$, where $\Phi_{t}$ denotes the corresponding quantum dynamical map. This construction is equivalent to Alice sending her states through

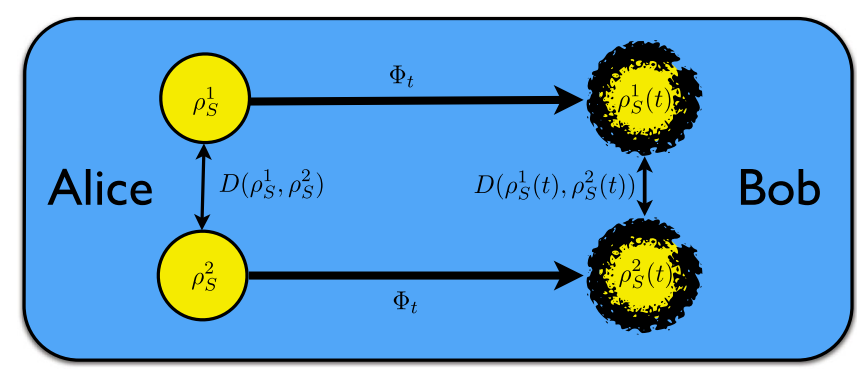

FIG. 2. The loss of distinguishability quantified by the trace distance $D$ between density matrices as a consequence of the action of a quantum dynamical map $\Phi_{t}$. Alice prepares two distinct states, but a dynamical map acts as a noisy channel, thus generally reducing the information available to Bob in order to distinguish among the states by performing measurements on the system only.

a noisy quantum channel described by the completely positive and trace preserving map $\Phi_{t}$. According to Eq. (28) the dynamics generally diminishes the trace distance and, therefore, the distinguishability of the states,

$$
D\left(\rho_{S}^{1}(t), \rho_{S}^{2}(t)\right) \leq D\left(\rho_{S}^{1}(0), \rho_{S}^{2}(0)\right),
$$

such that it will in general be harder for Bob to discriminate the states prepared by Alice, as illustrated schematically in Fig. 2. Thus, we can interpret any decrease of the trace distance $D\left(\rho_{S}^{1}(t), \rho_{S}^{2}(t)\right)$ as a loss of information from the open system into the environment. Conversely, if the trace distance $D\left(\rho_{S}^{1}(t), \rho_{S}^{2}(t)\right)$ increases, we say that information flows back from the environment into the open system.

This interpretation naturally leads to the following definition: A quantum process given by a family of quantum dynamical maps $\Phi_{t}$ is said to be Markovian if the trace distance $D\left(\rho_{S}^{1}(t), \rho_{S}^{2}(t)\right)$ corresponding to all pairs of initial states $\rho_{S}^{1}(0)$ and $\rho_{S}^{2}(0)$ decreases monotonically for all times $t \geq 0$. Quantum Markovian behavior thus means a continuous loss of information from the open system to the environment. Conversely, a quantum process is non-Markovian if there is an initial pair of states $\rho_{S}^{1}(0)$ and $\rho_{S}^{2}(0)$ such that the trace distance $D\left(\rho_{S}^{1}(t), \rho_{S}^{2}(t)\right)$ is nonmonotonic, i.e., starts to increase for some time $t>0$. If this happens, information flows from the environment back to the open system, which clearly expresses the presence of memory effects: Information contained in the open system is temporarily stored in the environment and comes back at a later time to influence the system. A crucial feature of this definition of quantum Markovian process is the fact that non-Markovianity can be directly experimentally assessed, provided one is able to perform tomographic measurement of different initial states at different times during the evolution. No prior information on the dynamical map $\Phi_{t}$ is required, apart from its very existence. We emphasize that according to this definition Markovianity or non-Markovianity is a property of the dynamical maps $\Phi_{t}$ describing the open system dynamics. In particular, this property depends on the Hamiltonian of Eq. (2) and can be changed by introducing, e.g., a timedependent control field. 
In order to explain in more detail the interpretation in terms of an information flow between system and environment let us define the quantities

$$
\mathcal{I}_{\text {int }}(t)=D\left(\rho_{S}^{1}(t), \rho_{S}^{2}(t)\right)
$$

and

$$
\mathcal{I}_{\text {ext }}(t)=D\left(\rho_{S E}^{1}(t), \rho_{S E}^{2}(t)\right)-D\left(\rho_{S}^{1}(t), \rho_{S}^{2}(t)\right)
$$

Here $\mathcal{I}_{\text {int }}(t)$ is the distinguishability of the open system states at time $t$, while $\mathcal{I}_{\text {ext }}(t)$ is the distinguishability of the total system states minus the distinguishability of the open system states at time $t$. In other words, $\mathcal{I}_{\text {ext }}(t)$ can be viewed as the gain in the state discrimination Bob could achieve if he were able to carry out measurements on the total system instead of measurements on the open system only. We can therefore interpret $\mathcal{I}_{\text {int }}(t)$ as the information inside the open system and $\mathcal{I}_{\text {ext }}(t)$ as the amount of information which lies outside the open system, i.e., as the information which is not accessible when only measurements on the open system can be performed. Obviously, we have $\mathcal{I}_{\text {int }}(t) \geq 0$ and $\mathcal{I}_{\text {ext }}(t) \geq 0$. Since the total system dynamics is unitary, the distinguishability of the total system states is constant in time. Moreover we have $D\left(\rho_{S E}^{1}(0), \rho_{S E}^{2}(0)\right)=D\left(\rho_{S}^{1}(0), \rho_{S}^{2}(0)\right)$ because, by assumption, the initial total states are uncorrelated with the same reduced environmental state. Hence, we obtain

$$
\mathcal{I}_{\text {int }}(t)+\mathcal{I}_{\text {ext }}(t)=\mathcal{I}_{\text {int }}(0)=\text { const. }
$$

Thus, initially there is no information outside the open system $\mathcal{I}_{\text {ext }}(0)=0$. If $\mathcal{I}_{\text {int }}(t)$ decreases, $\mathcal{I}_{\text {ext }}(t)$ must increase and vice versa, which clearly expresses the idea of the exchange of information between the open system and the environment illustrated in Fig. 3. Employing the properties of the trace distance one can derive the following general inequality (Laine, Piilo, and Breuer, 2010b) which holds for all $t \geq 0$ :

$$
\begin{aligned}
\mathcal{I}_{\text {ext }}(t) \leq & D\left(\rho_{S E}^{1}(t), \rho_{S}^{1}(t) \otimes \rho_{E}^{1}(t)\right) \\
& +D\left(\rho_{S E}^{2}(t), \rho_{S}^{2}(t) \otimes \rho_{E}^{2}(t)\right)+D\left(\rho_{E}^{1}(t), \rho_{E}^{2}(t)\right) .
\end{aligned}
$$

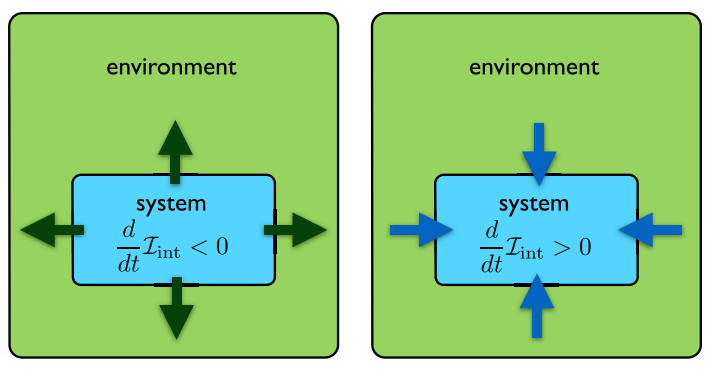

FIG. 3. The information flow between an open system and its environment according to Eq. (32). Left: The open system loses information to the environment, corresponding to a decrease of $\mathcal{I}_{\text {int }}(t)$ and Markovian dynamics. Right: Non-Markovian dynamics is characterized by a backflow of information from the environment to the system and a corresponding increase of $\mathcal{I}_{\text {int }}(t)$.
The right-hand side of this inequality consists of three terms: The first two terms provide measures for the correlations in the total system states $\rho_{S E}^{1,2}(t)$, given by the trace distance between these states and the products of their marginals. Note that these terms quantify both quantum and classical correlations of the total system states. The third term is the trace distance between the corresponding environmental states. Thus, when $\mathcal{I}_{\text {ext }}(t)$ increases over the initial value $\mathcal{I}_{\text {ext }}(0)=0$ system-environment correlations are built up or the environmental states become different, implying an increase of the distinguishability of the environmental states. This demonstrates that the corresponding decrease of the distinguishability $\mathcal{I}_{\text {int }}(t)$ of the open system states always has an impact on degrees of freedom which are inaccessible by measurements on the open system. Conversely, if $\mathcal{I}_{\text {int }}(t)$ starts to increase at some point of time $t$, the corresponding decrease of $\mathcal{I}_{\text {ext }}(t)$ implies that systemenvironment correlations must be present already at time $t$, or that the environmental states are different at this point of time.

Using this definition for non-Markovian dynamics one is naturally led to introduce the following measure for the degree of memory effects:

$$
\mathcal{N}(\Phi)=\max _{\rho_{S}^{1,2}} \int_{\sigma>0} d t \sigma(t)
$$

where

$$
\sigma(t) \equiv \frac{d}{d t} D\left(\Phi_{t} \rho_{S}^{1}, \Phi_{t} \rho_{S}^{2}\right)
$$

denotes the time derivative of the trace distance of the evolved pair of states. In Eq. (34) the time integral is extended over all intervals in which $\sigma(t)>0$, i.e., in which the trace distance increases with time, and the maximum is taken over all pairs of initial states $\rho_{S}^{1,2}$ of the open system's state space $\mathcal{S}\left(\mathcal{H}_{S}\right)$. Thus, $\mathcal{N}(\Phi)$ is a positive functional of the family of dynamical maps $\Phi$ which represents a measure for the maximal total flow of information from the environment back to the open system. By construction we have $\mathcal{N}(\Phi)=0$ if and only if the process is Markovian.

The maximization over all pairs of quantum states in Eq. (34) can be simplified substantially employing several important properties of the functional $\mathcal{N}(\Phi)$ and the convex structure of the set of quantum states. A certain pair of states $\rho_{S}^{1,2}$ is said to be an optimal state pair if the maximum in Eq. (34) is attained for this pair of states. Thus, optimal state pairs lead to the maximal possible backflow of information during their time evolution. One can show that optimal state pairs $\rho_{S}^{1,2}$ lie on the boundary of the state space, and are, in particular, always orthogonal (Wißmann et al., 2012). This is a quite natural result in view of the interpretation in terms of an information flow since orthogonality implies that $D\left(\rho_{S}^{1}, \rho_{S}^{2}\right)=1$, which shows that optimal state pairs have maximal initial distinguishability, corresponding to a maximal amount of initial information. An even more drastic simplification is obtained by employing the following equivalent representation (Liu et al., 2014): 


$$
\mathcal{N}(\Phi)=\max _{\rho \in \partial U\left(\rho_{0}\right)} \int_{\bar{\sigma}>0} d t \bar{\sigma}(t),
$$

where

$$
\bar{\sigma}(t) \equiv \frac{(d / d t) D\left(\Phi_{t} \rho, \Phi_{t} \rho_{0}\right)}{D\left(\rho, \rho_{0}\right)}
$$

is the time derivative of the trace distance at time $t$ divided by the initial trace distance. In Eq. (36) $\rho_{0}$ is a fixed point of the interior of the state space and $\partial U\left(\rho_{0}\right)$ is an arbitrary surface in the state space enclosing, but not containing $\rho_{0}$. The maximization is then taken over all points of such an enclosing surface. This representation often significantly simplifies the analytical, numerical, or experimental determination of the measure since it only involves a maximization over a single input state $\rho$. It is particularly advantageous if the open system dynamics has an invariant state and if this state is taken to be $\rho_{0}$, such that only one state of the pair evolves in time. Equation (36) may be called local representation for it shows that optimal state pairs can be found in any local neighborhood of any interior point of the state space. Furthermore, the representation reveals the universality of memory effects, namely, that for a non-Markovian dynamics memory effects can be observed everywhere in state space.

\section{Generalizing the trace distance measure}

There is an interesting generalization of this definition and quantification of non-Markovian quantum dynamics first suggested by Chruściński, Kossakowski, and Rivas (2011). Returning to the interpretation of the trace distance described in Sec. II.C.1, we may suppose that Alice prepares her quantum system in the states $\rho_{S}^{1}$ or $\rho_{S}^{2}$ with corresponding probabilities $p_{1}$ and $p_{2}$, where $p_{1}+p_{2}=1$. Thus, we assume that Alice gives certain weights to her states which need not be equal. In this case one can again derive an expression for the maximal probability for Bob to identify the state prepared by Alice (Helstrom, 1976):

$$
P_{\max }=\frac{1}{2}\left(1+\left\|p_{1} \rho_{S}^{1}-p_{2} \rho_{S}^{2}\right\|\right) .
$$

As follows from Eq. (38) that the quantity $\left\|p_{1} \rho_{S}^{1}-p_{2} \rho_{S}^{2}\right\|$ gives a bias in favor of the correct identification of the state prepared by Alice. We note that the operator

$$
\Delta=p_{1} \rho_{S}^{1}-p_{2} \rho_{S}^{2}
$$

is known as a Helstrom matrix (Helstrom, 1967), and Eq. (38) reduces to Eq. (27) in the unbiased case $p_{1}=p_{2}=1 / 2$. Note that the interpretation in terms of an information flow between system and environment still holds for the biased trace distance, as can be seen replacing in Eqs. (30) and (31) the trace distance by the norm of the Helstrom matrix.

Following this approach we can define a process to be Markovian if the function $\left\|\Phi_{t} \Delta\right\|$ decreases monotonically with time for all $p_{1,2}$ and all pairs of initial open system states $\rho_{S}^{1,2}$. Consequently, the extended measure for nonMarkovianity is then given by (Wißmann, Breuer, and Vacchini, 2015)

$$
\mathcal{N}(\Phi)=\max _{p_{i}, \rho_{S}^{i}} \int_{\sigma>0} d t \sigma(t)
$$

where $\sigma(t) \equiv(d / d t)\left\|\Phi_{t} \Delta\right\|$. This generalized definition of quantum non-Markovianity leads to several important conclusions. First, we note that also for the measure (40) optimal state pairs are orthogonal, satisfying $\left\|p_{1} \rho_{S}^{1}-p_{2} \rho_{S}^{2}\right\|=1$ and, therefore, corresponding to maximal initial distinguishability. The maximum in Eq. (40) can therefore be taken over all Hermitian (Helstrom) matrices $\Delta$ with unit trace norm. This leads to a local representation analogous to Eq. (36):

$$
\mathcal{N}(\Phi)=\max _{p_{i}, \rho_{S}^{2} \in \partial U\left(\rho_{S}^{1}\right)} \int_{\bar{\sigma}>0} d t \bar{\sigma}(t),
$$

where $\bar{\sigma}(t)=(d / d t)\left\|\Phi_{t} \Delta\right\| /\|\Delta\|$, and $\partial U\left(\rho_{S}^{1}\right)$ is again a closed surface which encloses a fixed point $\rho_{S}^{1}$ in the interior of the state space. This generalized measure does in general lead to different estimates of non-Markovianity and is positive even for quantum processes for which Eq. (34) is equal to zero. In particular, the generalized criterion detects the nonMarkovianity arising in a dynamics described by a uniform translation of states, neglected by the trace distance measure (Liu, Lu, and Wang, 2013).

Another important conclusion is obtained if we assume, as done in Sec. II.A.2, that $\Phi_{t}^{-1}$ exists. It can be shown (Wißmann, Breuer, and Vacchini, 2015) that under this condition the quantum process is Markovian if and only if $\Phi_{t}$ is $P$ divisible, which follows immediately from a theorem by Kossakowski (1972a, 1972b). The relationships between Markovianity and divisibility of the dynamical map are illustrated in Fig. 4, where also the situation in which the

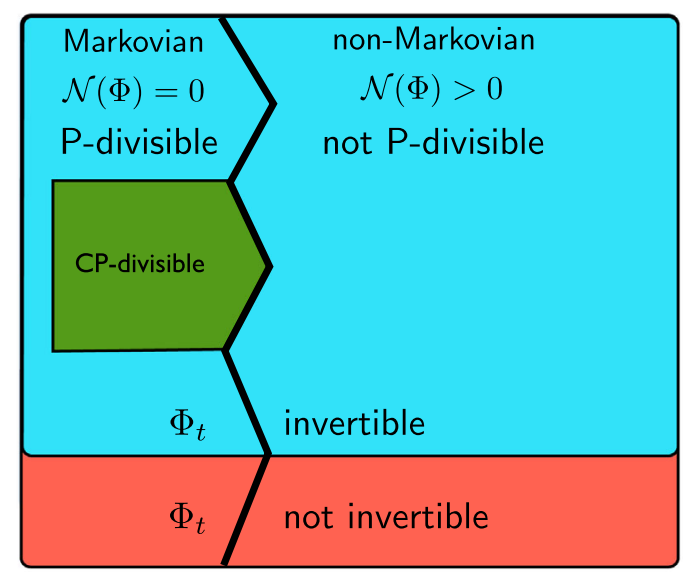

FIG. 4. The relations between the concepts of quantum Markovianity and divisibility of the family of quantum dynamical maps $\Phi_{t}$. The blue (light gray) area to the left of the thick zigzag line represents the set of Markovian quantum processes for which the inverse of the dynamical map exists, which is identical to the set of $P$-divisible processes. This set contains as a subset the set of $C P$-divisible processes (green). The blue (light gray) area to the right represents the non-Markovian processes which are therefore neither $C P$ nor $P$ divisible. The zigzag line dividing the two regions points to the fact that neither of them is convex. The red (dark gray) area marks the Markovian or non-Markovian processes for which the inverse of $\Phi_{t}$ does not exist. 
inverse of $\Phi_{t}$ as a linear map does not exist for all times has been considered. It is worth stressing that in keeping with the spirit of the approach, this generalized measure of nonMarkovianity, in addition to its clear connection with the mathematical property of $P$ divisibility of the dynamical map, can still be directly evaluated by means of experiments. More specifically, both the quantification of non-Markovianity according to the measure (34) and its generalization (40) can be obtained from the very same data.

\section{Connection between quantum and classical non-Markovianity}

The definition of quantum non-Markovianity of Sec. II.C.3 allows one to establish an immediate general connection to the classical definition as discussed by Wißmann, Breuer, and Vacchini (2015). Suppose as in Sec. II.A.2 that the inverse of the dynamical maps $\Phi_{t}$ exists such that we have the time-local quantum master equation (13), in which for simplicity we consider only the rates to be time dependent. Assume for simplicity that $\Phi_{t}$ maps operators which are diagonal in a fixed basis $\{|n\rangle\}$ to operators diagonal in the same basis. If $\rho_{S}(0)$ is an initial state diagonal in this basis, then $\rho_{S}(t)$ can be expressed at any time in the form

$$
\rho_{S}(t)=\sum_{n} P_{n}(t)|n\rangle\langle n|,
$$

where $P_{n}(t)$ denote the time-dependent eigenvalues. The quantum master equation then leads to the following equation of motion for the probabilities $P_{n}(t)$ :

$$
\frac{d}{d t} P_{n}(t)=\sum_{m}\left[W_{n m}(t) P_{m}(t)-W_{m n}(t) P_{n}(t)\right],
$$

where

$$
W_{n m}(t)=\sum_{i} \gamma_{i}(t)\left|\left\langle n\left|A_{i}\right| m\right\rangle\right|^{2}
$$

We observe that Eq. (43) has exactly the structure of a classical Pauli master equation (23). Therefore, Eq. (43) can be interpreted as a differential Chapman-Kolmogorov equation (22) for the conditional transition probability of a classical Markov process if and only if the rates (44) are positive. As seen a quantum process is Markovian if and only if the dynamics is $P$ divisible. According to Eq. (14) $P$ divisibility implies that the rates (44) are indeed positive. Thus, we conclude that any quantum Markovian process which preserves the diagonal structure of quantum states in a fixed basis leads to a classical Markovian jump process describing transitions between the eigenstates of the density matrix.

\section{Alternative approaches}

In Sec. II.C we considered an approach to quantum nonMarkovianity based on the study of the dynamical behavior of the distinguishability of states. This viewpoint directly addresses the issue of the experimental detection of quantum non-Markovian processes, which can be obtained by suitable quantum tomographic measurements. It further highlights a strong connection between quantum non-Markovianity and quantum information theory, or more specifically quantum information processing. A related approach, to be discussed in detail later, exploits the notion of $C P$ divisibility of a family of quantum dynamical maps, already defined in Sec. II.A.2 in order to introduce time-local master equations, and builds upon the analogy between the divisibility condition (12) and the Chapman-Kolmogorov equation (21).

Alternative approaches to the study of quantum nonMarkovian dynamics of open systems were also recently proposed. We try to briefly discuss those which are more closely related to the previous study, without any claim to properly represent the vast literature on the subject. For a review about recent results on quantum non-Markovianity see Rivas, Huelga, and Plenio (2014). The different approaches can to some extent be grouped according to whether they propose different divisibility properties of the quantum dynamical map (Wolf et al., 2008; Rivas, Huelga, and Plenio, 2010; Hou et al., 2011; Chruściński and Maniscalco, 2014; Hall et al., 2014), different quantifiers of the distinguishability between states (Lu, Wang, and Sun, 2010; Dajka, Łuczka, and Hänggi, 2011; Vasile, Maniscalco et al., 2011; Chruściński and Kossakowski, 2012; Wißmann, Leggio, and Breuer, 2013), or the study of other quantities, be they related to quantum information concepts or not, which might exhibit a monotonic or an oscillating behavior in time (Lu, Wang, and Sun, 2010; Luo, Fu, and Song, 2012; Bylicka, Chruściński, and Maniscalco, 2014; Fanchini et al., 2014; Haseli et al., 2014; Dhar, Bera, and Adesso, 2015).

\section{Quantum non-Markovianity and $\boldsymbol{C P}$ divisibility}

Let us first come back to the notion of divisible maps introduced in Sec. II.A.2, which we express in the form

$$
\Phi_{t, s}=\Phi_{t, \tau} \Phi_{\tau, s}, \quad t \geq \tau \geq s \geq 0
$$

where the maps have been defined according to Eq. (11). In the classical case considering the matrices whose elements are given by the conditional transition probabilities (18) according to $\left(\Lambda_{t, s}\right)_{x y}=T(x, t \mid y, s)$ the Chapman-Kolmogorov equation takes the form

$$
\Lambda_{t, s}=\Lambda_{t, \tau} \Lambda_{\tau, s}, \quad t \geq \tau \geq s \geq 0,
$$

where each $\Lambda_{t, s}$ is a stochastic matrix, which bears a strict relationship with Eq. (45), once one considers that positive maps are a natural quantum counterpart of classical stochastic matrices. Given the fact that in the classical case the Markov condition (17) entails the Chapman-Kolmogorov equation, and building on the conclusion drawn at the end of Sec. II.B.2, i.e., that an intrinsic characterization of memory effects has to be based solely on the dynamical maps, one is led to associate quantum Markovianity with $P$ divisibility. This observation reinforces the result obtained in Sec. II.C.3 relying on the study of the information flow as quantified by the generalized trace distance measure. Actually, similar to Sec. II.C.3 it can be shown that the $P$-divisibility condition (46) is equivalent to the monotonicity property of the function

$$
K\left(P_{1}^{1}(t), P_{1}^{2}(t)\right)=\sum_{x}\left|p_{1} P_{1}^{1}(x, t)-p_{2} P_{1}^{2}(x, t)\right|
$$


which provides a generalization of the Kolmogorov distance between two classical probability distributions $P_{1}^{1}(t)$ and $P_{1}^{2}(t)$ (Fuchs and van de Graaf, 1999). Therefore these two equivalent properties capture the feature of a classical Markovian process at the level of the one-point probability distribution, which is all we are looking for in order to obtain an intrinsic definition of quantum non-Markovianity.

Relying on the fact that in the theory of open quantum systems complete positivity suggests itself as a natural counterpart of positivity, as an alternative approach to quantum non-Markovianity it was proposed to identify quantum Markovian dynamics with those dynamics which are actually $C P$ divisible. This criterion was first suggested by Rivas, Huelga, and Plenio (2010). In order to check $C P$ divisibility of the time evolution one actually needs to know the transition maps $\Phi_{t, s}$ or the infinitesimal generator $\mathcal{K}_{t}$. Indeed given the maps $\Phi_{t, s}$ one can determine their complete positivity studying the associated Choi matrices, while as discussed in Sec. II.A $C P$ divisibility corresponds to positivity at all times of the rates $\gamma_{i}(t)$ appearing in Eq. (13). In order to quantify non-Markovianity as described by this criterion, different related measures have been introduced, relying on an estimate of the violation of positivity of the Choi matrix (Rivas, Huelga, and Plenio, 2010) or on various quantifiers of the negativity of the rates (Hall et al., 2014). In particular, Rivas, Huelga, and Plenio (2010) suggested to consider the following measure of non-Markovianity based on $C P$ divisibility:

$$
\mathcal{N}_{\mathrm{RHP}}(\Phi)=\int_{0}^{\infty} d t g(t)
$$

with

$$
g(t)=\lim _{\epsilon \rightarrow 0^{+}} \frac{\left\|\left(\Phi_{t+\epsilon, t} \otimes I\right)(|\Psi\rangle\langle\Psi|)\right\|-1}{\epsilon},
$$

where $|\Psi\rangle$ is a maximally entangled state between an open system and an ancilla.

A further feature which can be considered in this context, and which has been proposed by Lorenzo, Plastina, and Paternostro (2013) as another signature of non-Markovianity, is the dynamical behavior of the volume of the accessible states, according to their parametrization in the generalized Bloch representation, identifying nonMarkovianity with the growth of this volume, which also allows for a geometrical description. This study can be performed, e.g., upon knowledge of the rates appearing in the time-local master equation, and turns out to be a strictly weaker requirement for Markovianity with respect to $P$ divisibility (Chruściński and Wudarski, 2013).

\section{Monotonicity of correlations and entropic quantities}

Another line of thought about non-Markovianity of a quantum dynamics is based on the study of the behavior in time of quantifiers of correlations between the open system of interest and an ancilla system. In this respect both entanglement (Rivas, Huelga, and Plenio, 2014) and quantum mutual information (Luo, Fu, and Song, 2012) have been considered. In this setting one considers an ancilla system and studies the behavior in time of the entanglement or the mutual information of an initially correlated joint system-ancilla state. Given the fact that both quantities are nonincreasing under the local action of a completely positive trace preserving transformation, $C P$ divisibility of the time evolution would lead to a monotonic decrease of these quantities. Their nonmonotonic behavior can therefore be taken as a signature or a definition of quantum non-Markovianity. Actually, Rivas, Huelga, and Plenio (2014) considered $C P$ divisibility as the distinguishing feature of non-Markovianity, so that a revival of entanglement in the course of time is just interpreted as a witness of nonMarkovianity. Luo, Fu, and Song (2012) proposed instead failure of monotonicity in the loss of quantum mutual information as a new definition of non-Markovianity. In both cases one considers as an initial state a maximally entangled state between system and ancilla. The quantification of the effect is obtained by summing up the increases over time of the considered quantity. Still other approaches have connected non-Markovianity with nonmonotonicity of the quantum Fisher information, considered as a quantifier of the information flowing between system and environment. However, in this case the information flow is not directly linked to a distance on the space of states (Lu, Wang, and Sun, 2010). Most recently non-Markovianity has also been connected with the breakdown of monotonicity of the quantum interferometric power (Dhar, Bera, and Adesso, 2015). A comparative study of different non-Markovianity measures for an open system coupled to both a non-Markovian environment and a Markovian noise was carried out by Apollaro et al. (2014).

In the trace distance approach one studies the behavior of the statistical distinguishability between states, and relying on the introduction of the Helstrom matrix (Chruściński, Kossakowski, and Rivas, 2011), this notion can be directly related to a divisibility property of the quantum dynamical map. The generalization of the trace distance approach also allows for a stronger connection to a quantum information theory viewpoint. Indeed, one can consider the quantum dynamical maps as a collection of time-dependent channels. In this respect the natural question is how well Bob can recover information on the state prepared by Alice performing a measurement after a given time $t$. A proposal related to this viewpoint was considered by Bylicka, Chruściński, and Maniscalco (2014), where two types of capacity of a quantum channel, namely, the so-called entanglement-assisted classical capacity and the quantum capacity, were considered. While the quantum data processing inequality (Nielsen and Chuang, 2000) warrants monotonicity of these quantities provided the time evolution is $C P$ divisible, the lack of this monotonicity has been taken as a definition of non-Markovianity of the dynamics. The connection between non-Markovianity and the quantum data processing inequality was also recently studied by Buscemi and Datta (2014).

\section{MODELS AND APPLICATIONS OF NON-MARKOVIANITY}

In this section we first address the description of simple prototypical systems, for which one can exactly describe the non-Markovian features of the dynamics according to the concepts and measures introduced in Sec. II. In particular, we 
stress how a non-Markovian dynamics leads to a recovery of quantum features according to the notion of information backflow. In the second part we present applications of the introduced non-Markovianity measures to a number of more involved models illustrating how memory effects allow one to analyze their dynamics and can be related to characteristic properties of complex environments, thus suggesting to use the non-Markovianity of the open system dynamics as a quantum probe for features of the environment.

\section{A. Prototypical model systems}

We consider first a two-level system undergoing pure decoherence. This situation can be described by a timeconvolutionless master equation of the form (13) with a single decay channel and allows us to demonstrate in a simple manner how the properties of the environment and the systemenvironment coupling strength influence the transition from a Markovian to a non-Markovian dynamics for the open quantum system. A similar analysis is performed for a dynamics which also takes into account dissipative effects, further pointing to the phenomenon of the failure of divisibility of the quantum dynamical map. We also discuss a dynamics driven by different decoherence channels, which allows us to better discriminate between different approaches to the description of non-Markovian behavior. Finally, we present some results on the non-Markovian dynamics of the spin-boson model.

\section{Pure decoherence model}

Let us start considering a microscopic model for a pure decoherence dynamics which is amenable to an exact solution and describes a single qubit interacting with a bosonic reservoir. The total Hamiltonian can be written as in Eq. (2) with the system Hamiltonian $H_{S}$ and the environmental Hamiltonian $H_{E}$ given by

$$
H_{S}=\frac{1}{2} \omega_{0} \sigma_{z}, \quad H_{E}=\sum_{k} \omega_{k} a_{k}^{\dagger} a_{k},
$$

where $\omega_{0}$ is the energy difference between ground state $|0\rangle$ and excited state $|1\rangle$ of the system, $\sigma_{z}$ denotes a Pauli matrix, and $a_{k}$ and $a_{k}^{\dagger}$ are annihilation and creation operators for the bosonic reservoir mode labeled by $k$ with frequency $\omega_{k}$, obeying the commutation relations $\left[a_{k}, a_{k^{\prime}}^{\dagger}\right]=\delta_{k k^{\prime}}$. The interaction term is taken to be

$$
H_{I}=\sum_{k} \sigma_{z}\left(g_{k} a_{k}+g_{k}^{*} a_{k}^{\dagger}\right)
$$

with coupling constants $g_{k}$. Considering a factorized initial condition with the environment in a thermal state at inverse temperature $\beta$ the model can be exactly solved leading to a quantum dynamical map $\Phi_{t}$ which leaves the populations invariant and modifies the off-diagonal matrix elements according to

$$
\rho_{11}(t)=\rho_{11}(0), \quad \rho_{10}(t)=G(t) \rho_{10}(0),
$$

where $\rho_{i j}(t)=\left\langle i\left|\rho_{S}(t)\right| j\right\rangle$ denote the elements of the interaction picture density matrix $\rho_{S}(t)$. The function $G(t)$ is often called decoherence function and in the present case it is real since we are working in the interaction picture. It can be expressed in the form

$G(t)=\exp \left[-\int_{0}^{\infty} d \omega J(\omega) \operatorname{coth}\left(\frac{\beta \omega}{2}\right) \frac{1-\cos (\omega t)}{\omega^{2}}\right]$,

where we introduced the spectral density $J(\omega)=$ $\sum_{k}\left|g_{k}\right|^{2} \delta\left(\omega-\omega_{k}\right)$ which keeps track of the features of the environment relevant for the reduced system description, containing information both on the environmental density of the modes and on how strongly the system couples to each mode. It can be shown that the interaction picture operator $\rho_{S}(t)$ obeys a master equation of the form (13) with a single Lindblad operator,

$$
\frac{d}{d t} \rho_{S}(t)=\gamma(t)\left[\sigma_{z} \rho_{S}(t) \sigma_{z}-\rho_{S}(t)\right]
$$

where the time-dependent decay rate $\gamma(t)$ is related to the decoherence function $G(t)$ by

$$
\gamma(t)=-\frac{1}{G(t)} \frac{d}{d t} G(t)
$$

Note that for a generic microscopic or phenomenological decoherence model the decoherence function is in general a complex quantity even in the interaction picture, and in this case the modulus of the function should be considered in Eq. (55). The map $\Phi_{t}$ is completely positive since $G(t) \leq 1$ which is equivalent to the positivity of the time integral of the decay rate $\Gamma(t)=\int_{0}^{t} d t^{\prime} \gamma\left(t^{\prime}\right)$. In order to discuss the nonMarkovianity of the obtained time evolution as discussed in Secs. II.C and II.D.1 we consider the divisibility property of the time evolution. The master equation (54) has a single channel with decay rate $\gamma(t)$ and thanks to the strict positivity of the decoherence function $\Phi_{t}^{-1}$ always exists. However, according to the criterion given by Eq. (14), $P$ divisibility fails at times when the decoherence rate becomes negative, which is exactly when $C P$ divisibility is lost. This is generally true when one has a master equation in the time-local form (13) with a single Lindblad operator, since in this case the condition for $C P$ divisibility, given by the requirement of positivity of the decay rate $\gamma(t)$, coincides with Eq. (14) for $P$ divisibility.

The trace distance between time evolved states corresponding to the initial conditions $\rho_{S}^{1,2}$ is given according to Eq. (26) by

$$
D\left(\Phi_{t} \rho_{S}^{1}, \Phi_{t} \rho_{S}^{2}\right)=\sqrt{a^{2}+G^{2}(t)|b|^{2}}
$$

where $a=\rho_{11}^{1}-\rho_{11}^{2}$ and $b=\rho_{10}^{1}-\rho_{10}^{2}$ denote the difference of the populations and of the coherences of the initial states, respectively. Its time derivative corresponding to the quantity (35) in terms of which the non-Markovianity measure can be constructed then reads 


$$
\sigma(t)=\frac{G(t)|b|^{2}}{\sqrt{a^{2}+G^{2}(t)|b|^{2}}} \frac{d}{d t} G(t) .
$$

The nonmonotonicity of the trace distance which is used as a criterion for non-Markovianity in Sec. II.C.2 is therefore determined by the nonmonotonic behavior of the decoherence function. The dependence of the non-Markovianity of the model on the spectral density has been studied by Addis et al. (2014) and Guarnieri, Smirne, and Vacchini (2014), and mainly depends on the low frequency behavior of the spectral density.

In order to evaluate the measure (34) one has to maximize over all pairs of initial states. As shown by Wißmann et al. (2012) and discussed in Sec. II.C.2 the latter can be restricted to orthogonal state pairs. For the case at hand it follows from Eq. (57) that the maximum is obtained for antipodal points on the equator of the Bloch sphere, so that $a=0$ and $|b|=1$. The non-Markovianity measure (34) is then given by

$$
\mathcal{N}(\Phi)=\sum_{k}\left[G\left(t_{k}^{f}\right)-G\left(t_{k}^{i}\right)\right]
$$

where $t_{k}^{i}$ and $t_{k}^{f}$ denote initial and final points of the $k$ th time interval in which $G(t)$ increases. Since the increase of $G(t)$ coincides with the negativity of the decoherence rate according to Eq. (55), the growth of the trace distance is in this case equivalent to breaking both $P$ divisibility and $C P$ divisibility. Moreover, the generalized trace distance measure considered in Sec. II.C.3 leads to the same expression (58) for nonMarkovianity.

The measure for non-Markovianity of Eq. (48) based on $C P$ divisibility takes in this case the simple expression

$$
\mathcal{N}_{\mathrm{RHP}}(\Phi)=-2 \int_{\gamma<0} d t \gamma(t),
$$

which is the integral of the decoherence rate in the time intervals in which it becomes negative. An example of an experimental realization of a pure decoherence model is considered in Sec. IV.C.1, where the decoherence function is determined by the interaction between the polarization and the frequency degrees of freedom of a photon. In this case the decoherence function is, in general, complex valued, so that the previous formulas hold with $G(t)$ replaced by $|G(t)|$. The geometric measure discussed in Sec. II.D.1, which connects non-Markovian behavior with the growth in time of the volume of accessible states, also leads to the same signature for non-Markovianity: The volume can again be identified with the decoherence function $G(t)$ and therefore as soon as $G(t)$ increases the volume grows.

As discussed, in a simple decoherence model nonMarkovianity can be identified with the revival of coherences of the open system, corresponding to a backflow of information from the environment to the system. In this situation the time-local master equation (54) describing the dynamics exhibits just a single channel, so that all discussed criteria of non-Markovianity coincide.

\section{Two-level system in a dissipative environment}

We now consider an example of dissipative dynamics in which the system-environment interaction influences both coherences and populations of a two-state system. The environment is still taken to be a bosonic bath, so that the free contributions to the Hamiltonian are again given by Eq. (50), but one considers an interaction term in the rotating wave approximation given by

$$
H_{I}=\sum_{k}\left(g_{k} \sigma_{+} a_{k}+g_{k}^{*} \sigma_{-} a_{k}^{\dagger}\right)
$$

where $\sigma_{-}=|0\rangle\langle 1|$ and $\sigma_{+}=|1\rangle\langle 0|$ are the lowering and raising operators of the system. Such an interaction describes, e.g., a two-level atom in a lossy cavity. For a factorized initial state with the environment in the vacuum state, this model is again exactly solvable, thanks to the conservation of the number of excitations. The quantum dynamical map $\Phi_{t}$ transforms populations and coherences according to

$$
\rho_{11}(t)=|G(t)|^{2} \rho_{11}(0), \quad \rho_{10}(t)=G(t) \rho_{10}(0)
$$

where the decoherence function is a complex function determined by the spectral density $J(\omega)$ of the model. In particular, denoting by $f\left(t-t_{1}\right)$ the two-point correlation function of the environment corresponding to the Fourier transform of the spectral density, the function $G(t)$ is determined as the solution of the integral equation

$$
\frac{d}{d t} G(t)=-\int d t_{1} f\left(t-t_{1}\right) G\left(t_{1}\right)
$$

with initial condition $G(0)=1$. Also in this case it is possible to write down the exact master equation for the density matrix $\rho_{S}(t)$ in the form of Eq. (13), which in the interaction picture reads (Breuer, Kappler, and Petruccione, 1999)

$$
\begin{aligned}
\frac{d}{d t} \rho_{S}(t)=- & \frac{i}{4} S(t)\left[\sigma_{z}, \rho_{S}\right] \\
& +\gamma(t)\left[\sigma_{-} \rho_{S}(t) \sigma_{+}-\frac{1}{2}\left\{\sigma_{+} \sigma_{-}, \rho_{S}(t)\right\}\right],
\end{aligned}
$$

where the time-dependent Lamb shift is given by $S(t)=-2 \Im[\dot{G}(t) / G(t)]$, and the decay rate can be written as $\gamma(t)=-2 \Re[\dot{G}(t) / G(t)]$, or equivalently

$$
\gamma(t)=-\frac{2}{|G(t)|} \frac{d}{d t}|G(t)|
$$

The analysis of non-Markovianity in the present model closely follows the discussion in Sec. III.A. 1 due to the crucial fact that the master equation (63) still has a single Lindblad operator. The expression of the trace distance is determined by the decoherence function and, with the same notation as in Eq. (56), reads

$$
D\left(\Phi_{t} \rho_{S}^{1}, \Phi_{t} \rho_{S}^{2}\right)=|G(t)| \sqrt{|G(t)|^{2} a^{2}+|b|^{2}},
$$


so that one has the time derivative

$$
\sigma(t)=\frac{2|G(t)|^{2} a^{2}+|b|^{2}}{\sqrt{|G(t)|^{2} a^{2}+|b|^{2}}} \frac{d}{d t}|G(t)| .
$$

Complete positivity of the map is ensured by positivity of the integrated decay rate $\Gamma(t)$, while the trace distance shows a nonmonotonic behavior when the modulus of the decoherence function grows with time.

A typical expression for the spectral density is given by a Lorentzian

$$
J(\omega)=\gamma_{0} \lambda^{2} / 2 \pi\left[\left(\omega_{0}+\Delta-\omega\right)^{2}+\lambda^{2}\right]
$$

of width $\lambda$ and centered at a frequency detuned from the atomic frequency $\omega_{0}$ by an amount $\Delta$, while the rate $\gamma_{0}$ quantifies the strength of the system-environment coupling. Let us first discuss the case when the qubit is in resonance with the central frequency of the spectral density, so that one has a vanishing detuning $\Delta=0$. The decoherence function then takes the form

$$
G(t)=e^{-\lambda t / 2}\left[\cosh \left(\frac{d t}{2}\right)+\frac{\lambda}{d} \sinh \left(\frac{d t}{2}\right)\right]
$$

where $d=\sqrt{\lambda^{2}-2 \gamma_{0} \lambda}$. For weak couplings, corresponding to $\gamma_{0}<\lambda / 2$, the decoherence function $G(t)$ is a real, monotonically decreasing function so that Eq. (66) is always negative and the dynamics is Markovian. In this case, as discussed in Sec. II.A.2, the map is invertible and since the time-convolutionless form of the master equation has just a single channel, it is at the same time $P$ and $C P$ divisible. Note that in the limit $\gamma_{0} \ll \lambda / 2$ the rate $\gamma(t)$ becomes time independent, $\gamma=\gamma_{0}$, such that the master equation (63) is of the Lindblad form and leads to a Markovian semigroup. However, for larger values of the coupling between system and environment, namely, for $\gamma_{0}>\lambda / 2$, the function $|G(t)|$ displays an oscillatory behavior leading to a nonmonotonic time evolution of the trace distance and, hence, to nonMarkovian dynamics. Thus, the trace distance measure of Eq. (34) is zero for $\gamma_{0}<\lambda / 2$ and starts at the threshold $\gamma_{0}=$ $\lambda / 2$ to increase continuously to positive values (Laine, Piilo, and Breuer, 2010a). It is interesting to note that the transition point $\gamma_{0}=\lambda / 2$ exactly coincides with the point where the perturbation expansion of the time-convolutionless master equation (63) breaks down. We also remark that for this model optimal state pairs still correspond to antipodal points of the equator of the Bloch sphere (Xu, Yang, and Feng, 2010), and that the criterion for non-Markovianity based on the Helstrom matrix considered in Sec. II.C.3 leads to the same results.

In the parameter regime $\gamma_{0}>\lambda / 2$ the map $\Phi_{t}$ defined by Eq. (61) is no longer invertible for all times due to the existence of zeros of the decoherence function. Thus, the model provides an example for a family of maps lying in the lower (red) region depicted in Fig. 4. Physically, the twolevel system reaches the ground state before memory effects revive the coherences and the population of the upper state. Indeed, also in this case non-Markovianity can be traced back to the revival of populations and coherences, corresponding to a backflow of information from the environment to the system. Since the inverse of $\Phi_{t}$ does not exist for all times, the criterion based on $C P$ divisibility cannot strictly speaking be applied. This is reflected by the fact that the corresponding measure, taking in this case again Eq. (59), jumps from zero to infinity.

In the nonresonant case $\Delta \neq 0$, one can observe a transition from Markovian to non-Markovian dynamics for a fixed value of the coupling in the weak coupling regime by increasing the detuning $\Delta$. In this case the map is invertible for all times, since the decay rate $\gamma(t)$ is finite for all times, so that also the measure (48) remains well defined. Again, the regions of nonMarkovianity coincide for all approaches since the constraint in order to have $C P$ or $P$ divisibility is the same. The pairs of states maximizing the expression of the measure (34) are, however, now given by the projections onto the excited and ground states. This shows how the optimal pair, which is always given by orthogonal states, depends on the actual expression for the decoherence function. For this case the derivative of the trace distance (66) takes the simple form $\sigma(t)=-\gamma(t) \exp [-\Gamma(t)]$ with $\Gamma(t)=\int_{0}^{t} \gamma\left(t^{\prime}\right) d t^{\prime}$, which puts into evidence the direct connection between the direction of information flow and the sign of the decay rate.

\section{Single qubit with multiple decoherence channels}

In the preceding examples we showed that for a master equation in the time-convolutionless form (13) with a single Lindblad operator $A(t)$ all the different considered criteria for non-Markovianity coincide. It is therefore of interest to discuss an example in which one has multiple decoherence channels with generally different rates. As we shortly see, such a model allows one to discriminate among the different definitions of non-Markovian dynamics. To this end, we take a phenomenological approach and following Vacchini (2012), Chruściński and Wudarski (2013), and Chruściński and Maniscalco (2014) we consider for a two-level system the master equation

$$
\frac{d}{d t} \rho_{S}(t)=\frac{1}{2} \sum_{i=1}^{3} \gamma_{i}(t)\left[\sigma_{i} \rho_{S}(t) \sigma_{i}-\rho_{S}(t)\right]
$$

where $\sigma_{i}$ with $i=x$, and $y$ and $z$ denote the three Pauli operators. Considering, e.g., the decoherence dynamics of a spin-1/2 particle in a complex environment the rates $\gamma_{i}(t)$ would correspond to generally time-dependent transversal and longitudinal decoherence rates. The dynamical map corresponding to Eq. (69) can be exactly worked out and is given by the random unitary dynamics

$$
\Phi_{t}\left(\rho_{S}\right)=\sum_{i=0}^{3} p_{i}(t) \sigma_{i} \rho_{S} \sigma_{i}
$$

where $\sigma_{0}$ denotes the identity operator and the coefficients $p_{i}(t)$ summing up to 1 are determined from the decoherence rates. Introducing the quantities $A_{i j}(t)=\exp \left[-\Gamma_{i}(t)-\Gamma_{j}(t)\right]$, where as usual we have denoted by $\Gamma_{k}(t)$ the time integral of the $k$ th decay rate, the coefficients $p_{i}(t)$ take the explicit form $p_{0,1}=(1 / 4)\left[1 \pm A_{12}(t) \pm A_{13}(t)+A_{23}(t)\right]$ and $p_{2,3}=$ $(1 / 4)\left[1 \mp A_{12}(t) \pm A_{13}(t)-A_{23}(t)\right]$. These equations show 
that the dynamical map actually depends on the sum of the integrals of the decay rates. According to its explicit expression the map $\Phi_{t}$ is completely positive provided the coefficients $p_{i}(t)$ remain positive, in which case they can be interpreted as a probability distribution and thus characterize the random unitary dynamics (70). Note that this can be the case even if a decoherence rate stays negative at all times as in Vacchini et al. (2011) and Hall et al. (2014).

As explained in Sec. II.A.2, the map is $C P$ divisible when all of the decoherence rates remain positive for all $t \geq 0$, so that as soon as at least one of the rates becomes negative the measure (48) becomes positive indicating a non-Markovian behavior. However, the condition (14) for having $P$ divisibility is weaker. Indeed, in order to have $P$-divisible dynamics only the sum of all pairs of distinct decoherence rates has to remain positive, i.e., $\gamma_{i}(t)+\gamma_{j}(t) \geq 0$ for all $j \neq i$. The same constraint warrants monotonicity in time of the behavior of the trace distance, so that also in this case the measure (34) of nonMarkovianity and its generalized version (40) based on the Helstrom matrix and corresponding to $P$ divisibility lead to the same result. In the characterization of non-Markovianity based on the backflow of information from the environment to the system memory effects therefore appear only whenever the sum of at least a pair of decoherence rates becomes negative. To better understand how this fact can be related to the dynamics of the system, one can notice (Chruściński and Maniscalco, 2014) that the Bloch vector components $\left\langle\sigma_{i}(t)\right\rangle$, according to Eq. (69), obey

$$
\frac{d}{d t}\left\langle\sigma_{i}(t)\right\rangle=-\frac{1}{T_{i}(t)}\left\langle\sigma_{i}(t)\right\rangle
$$

where the relaxation times are given by $T_{i}(t)=\left[\gamma_{j}(t)+\right.$ $\left.\gamma_{k}(t)\right]^{-1}$ (with all three indices taken to be distinct) and correspond to experimentally measurable quantities. The appearance of non-Markovianity and therefore the failure of $P$ divisibility is thus connected to negative relaxation rates, corresponding to a rebuild of quantum coherences. For the present model one can also easily express the condition leading to a growth of the volume of accessible states within the Bloch sphere. In fact, this volume of accessible states is proportional to $\exp \left[-\sum_{i=1}^{3} \Gamma_{i}(t)\right]$. As a consequence, the dynamics according to the geometric criterion by Lorenzo, Plastina, and Paternostro (2013) is Markovian if and only if $\sum_{i=1}^{3} \gamma_{i}(t) \geq 0$ for $t \geq 0$, which is a strictly weaker criterion with respect to either $P$ or $C P$ divisibility.

While in the examples considered the non-Markovianity measure (34) based on the trace distance and its generalization (40) based on the Helstrom matrix agree in the indication of non-Markovian dynamics, a situation in which these criteria are actually different was considered by Chruściński, Kossakowski, and Rivas (2011) and Wißmann, Breuer, and Vacchini (2015).

\section{Spin-boson model}

Finally, we briefly discuss a further paradigmatic model for dissipative quantum dynamics with many applications, namely, the spin-boson model (Leggett et al., 1987). The system and the environmental Hamiltonian are again given by
Eq. (50), while the interaction Hamiltonian has the nonrotating-wave structure

$$
H_{I}=\sum_{k} \sigma_{x}\left(g_{k} a_{k}+g_{k}^{*} a_{k}^{\dagger}\right)
$$

For this model the quantum dynamical map cannot be obtained analytically, so that one has to resort to approximate or numerical solutions. If the system-environment coupling is weak one can consider contributions up to second order obtaining a time-local master equation of the form (13), in which the time-dependent coefficients $\gamma_{i}(t)$ are given by suitable two-point correlation functions of the environment. The latter are best expressed by means of the spectral density $J(\omega)$ of the environmental modes, and the dynamics turns out to be determined by the so-called noise and dissipation kernels $D_{1}(\tau)$ and $D(\tau)$ which take the form (Breuer and Petruccione, 2002)

$$
\begin{gathered}
D_{1}(\tau)=2 \int_{0}^{\infty} d \omega J(\omega) \operatorname{coth}\left(\omega / 2 k_{\mathrm{B}} T\right) \cos (\omega \tau), \\
D(\tau)=2 \int_{0}^{\infty} d \omega J(\omega) \sin (\omega \tau) .
\end{gathered}
$$

Figure 5 shows the non-Markovianity measure $\mathcal{N}(\Phi)$ for this model obtained from numerical simulations of the corresponding second-order time-convolutionless master equation, using an Ohmic spectral density of Lorentz-Drude shape with cutoff frequency $\Omega$, reservoir temperature $T$, and a fixed small coupling strength of size $\gamma=0.1 \omega_{0}$ (Clos and Breuer, 2012). As can be seen from the figure the dynamics is strongly nonMarkovian for both small cutoff frequencies and small temperatures (note the logarithmic scale of the color bar).

The emergence of a Markovian region within the nonMarkovian regime for small cutoffs and temperatures can be understood in terms of a resonance between the transition frequency and the maximum of the effective, temperaturedependent spectral density of the environmental modes

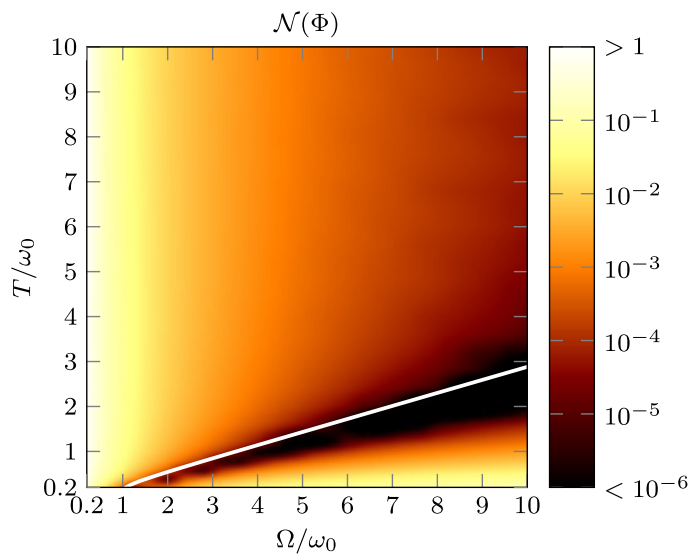

FIG. 5. The non-Markovianity measure $\mathcal{N}(\Phi)$ defined by Eq. (34) for the spin-boson model as a function of temperature $T$ and cutoff frequency $\Omega$ in units of the transition frequency $\omega_{0}$. The white curve given by Eq. (76) describes the points at which $\omega_{0}$ is exactly in resonance with the maximum of the effective spectral density (75). From Clos and Breuer, 2012. 


$$
J_{\text {eff }}(\omega)=J(\omega) \operatorname{coth}\left(\omega / 2 k_{\mathrm{B}} T\right)
$$

appearing in the noise kernel $D_{1}(\tau)$. Indeed, as can be seen from Fig. 5 the diagonal black (Markovian) region is well described by the white curve in the $(\Omega, T)$ plane which is defined by

$$
\left.\frac{\partial J_{\text {eff }}}{\partial \omega}\right|_{\omega=\omega_{0}}=0
$$

corresponding to the points at which the transition frequency $\omega_{0}$ of the system is exactly in resonance with the maximum of the effective spectral density.

\section{B. Applications of quantum non-Markovianity}

In Sec. III.A we illustrated quantum non-Markovian behavior by means of simple model systems. The goal of this section is to demonstrate that memory effects and their control also open new perspectives for applications. In fact, understanding various aspects of non-Markovianity makes it possible to develop more refined tools for reservoir engineering and to use memory effects as an indicator for the presence of typical quantum features. Moreover, it becomes possible to develop schemes where a small open system is used as a quantum probe detecting characteristic properties of the complex environment it is interacting with. Here we present a few examples from the recent literature illustrating these points.

\section{Analysis and control of non-Markovian dynamics}

We first consider the model of a spin- $1 / 2$ particle with spin operator $\mathbf{s}_{0}$ coupled to a chain of $N$ spin- $1 / 2$ particles with spin operators $\mathbf{s}_{n}(n=1,2, \ldots, N)$, which was investigated by Apollaro et al. (2011). The system Hamiltonian is given by $H_{S}=-2 h_{0} s_{0}^{z}$, where $h_{0}$ denotes a local field acting on the system spin $\mathbf{s}_{0}$. The environmental Hamiltonian represents an $X X$-Heisenberg spin chain with nearest neighbor interactions of strength $J$ in a transverse magnetic field $h$ :

$$
H_{E}=-2 J \sum_{n=1}^{N-1}\left(s_{n}^{x} s_{n+1}^{x}+s_{n}^{y} s_{n+1}^{y}\right)-2 h \sum_{n=1}^{N} s_{n}^{z} .
$$

The interaction Hamiltonian takes the form

$$
H_{I}=-2 J_{0}\left(s_{0}^{x} s_{1}^{x}+s_{0}^{y} s_{1}^{y}\right),
$$

describing an energy-exchange interaction between the system spin $\mathbf{s}_{0}$ and the first spin $\mathbf{s}_{1}$ of the chain with coupling strength $J_{0}$. This model leads to a rich dynamical behavior of the system spin and allows one to demonstrate not only the impact of the system-environment interaction, but also how the open system dynamics changes when manipulating the interactions between the constituents of the environment and their local properties.

It can be shown analytically that optimal state pairs for the trace distance measure correspond to antipodal points on the equator of the surface of the Bloch sphere. Moreover, when the local fields of the system and environmental sites are equal, $h=h_{0}$, one can derive an analytic expression for the rate of change $\sigma(t)$ of the trace distance [see Eq. (35)] for optimal state pairs:

$$
\sigma(t)=-(2 / t) \operatorname{sgn}\left[\mathcal{J}_{1}(2 t)\right] \mathcal{J}_{2}(2 t),
$$

where sgn is the sign function, and $\mathcal{J}_{1}$ and $\mathcal{J}_{2}$ denote the Bessel functions of order 1 and 2 , respectively. In the physically more interesting case $h \neq h_{0}$ the trace distance measure $\mathcal{N}(\Phi)$ [see Eq. (34)] has to be determined numerically. Let us first consider the case, where the system local field is zero, $h_{0}=0$, the couplings between the spins are equal, $J=J_{0}$, and we tune the ratio $h / J$ of the local field with respect to the spin-spin interaction strength. It turns out that the open system is then Markovian only at the point $h / J=1 / 2$, i.e., when the strength of the local field is half of the coupling between the spins. For all other values of $h / J$ the open system dynamics displays memory effects with $\mathcal{N}(\Phi)>0$. Thus, the point of Markovianity separates two regions where memory effects are present. This behavior persists for $h_{0} \neq 0$ : Denoting the detuning between the local fields by $\delta=h-h_{0}$ and still keeping $J=J_{0}$, the point of Markovianity occurs at $\delta h / J=1 / 2$. On both sides of this point the system again exhibits memory effects, but interestingly their properties are different. For $\delta h / J<1 / 2$ the system approaches a steady state which is independent of the initial state. On the other hand, for $\delta h / J>1 / 2$ the trace distance does not decay to zero asymptotically in time. This feature can be interpreted as information trapping since it implies that the distinguishability of states does not vanish asymptotically. We also remark that the origin of memory effects and the differences in the dynamical behavior can be understood in more detail by studying the spectrum of the total Hamiltonian (Apollaro et al., 2011).

As our second example we discuss applications to a physical system which has been studied extensively during the last 20 years, namely, Bose-Einstein condensates (BECs) which can be manipulated with high precision by current technologies and thus provide means for environment engineering (Dalfovo et al., 1999; Pitaevskii and Stringari, 2003; Pethick and Smith, 2008). Following Haikka et al. (2011) we consider an impurityBEC system, where the Markovian to non-Markovian transition can be controlled by tuning the properties of the BEC. An impurity atom is trapped within a double-well potential, where the left $(|L\rangle)$ and right $(|R\rangle)$ states represent the two qubit states. The BEC, which constitutes the environment of the qubit, is trapped in a harmonic potential. The Hamiltonian for the total impurity-BEC system reads

$$
\begin{aligned}
H= & \sum_{\mathbf{k}} E_{\mathbf{k}} c_{\mathbf{k}}^{\dagger} c_{\mathbf{k}}+\sum_{\mathbf{k}}\left(\xi_{\mathbf{k}} c_{\mathbf{k}}^{\dagger}+\xi_{\mathbf{k}}^{*} c_{\mathbf{k}}\right) \\
& +\sum_{\mathbf{k}} \sigma_{z}\left(g_{\mathbf{k}} c_{\mathbf{k}}^{\dagger}+g_{\mathbf{k}}^{*} c_{\mathbf{k}}\right) .
\end{aligned}
$$

Here $E_{\mathbf{k}}$ denotes the energy of the Bogoliubov mode $c_{\mathbf{k}}$ of the condensate, $\sigma_{z}=|R\rangle\langle R|-| L\rangle\langle L|$, while $g_{\mathbf{k}}$ and $\xi_{\mathbf{k}}$ describe the impurity-BEC and the intra-BEC couplings, respectively.

For a background $\mathrm{BEC}$ at zero temperature the open qubit dynamics is described by a dephasing master equation of the form of Eq. (54) and it turns out that the decoherence rate $\gamma(t)$ can be tuned by changing the interwell distance, and by 
controlling the dimensionality and the interaction strength (scattering length) of the BEC. Detailed studies demonstrate that for a 3D gas an increase of the well separation and of the intra-environment interaction leads to an increase of the nonMarkovianity measure for the impurity dynamics. Moreover, the dimensionality of the environment influences the emergence of memory effects. Studying the Markovian to non-Markovian transition as a function of the intraenvironment interaction strength shows that a 3D environment is the most sensitive. The Markovian-non-Markovian crossover occurs in 3D for weaker values of the scattering length than in 1D and 2D environments, and a 1D environment requires the largest scattering length for the crossover. This behavior can be ultimately traced back to the question of how the dimensionality of the environment influences the spectral density which governs the open system dynamics. In general, for small frequencies the spectral density shows a power law behavior $J(\omega) \propto \omega^{s}$. The spectral density is called sub-Ohmic, Ohmic, and super-Ohmic for $s<1, s=1$, and $s>1$, respectively. For a 3D gas, even without interactions within the gas, the spectral density has super-Ohmic character. Introducing interactions within the environment then makes this character stronger and thereby the $3 \mathrm{D}$ gas is most sensitive to the Markovian to non-Markovian transition. In contrast, for the 1D gas with increasing scattering length the spectral density first changes from sub-Ohmic to Ohmic and finally to super-Ohmic which allows the appearance of memory effects.

\section{Open systems as non-Markovian quantum probes}

In addition to detecting, quantifying, and controlling memory effects, it turns out to be fruitful to ask whether the presence or absence of such effects in the dynamics of an open quantum system allows one to obtain important information about characteristic features of a complex environment the open system is interacting with. To illustrate this point we present a physical scenario, where Markovian behavior indicates the presence of a quantum phase transition in a spin environment (Sachdev, 2011). Consider a system qubit, with states $|g\rangle$ and $|e\rangle$, which interacts with an environment described by a one-dimensional Ising model in a transverse field (Quan et al., 2006). The Hamiltonians of the environment and the qubit-environment interaction are given by

$$
\begin{gathered}
H_{E}=-J \sum_{j}\left(\sigma_{j}^{z} \sigma_{j+1}^{z}+\lambda \sigma_{j}^{x}\right), \\
H_{I}=-J \delta|e\rangle\langle e| \sum_{j} \sigma_{j}^{x},
\end{gathered}
$$

where $J$ is a microscopic energy scale, $\lambda$ is a dimensionless coupling constant describing the strength of the transverse field, and $\sigma_{j}^{x, z}$ are Pauli spin operators. The system qubit is coupled with strength $\delta$ to all environmental spins. The model yields pure dephasing dynamics described by a decoherence function $G(t)$ which, for a pure environmental initial state $|\Phi\rangle$, is given by

$$
G(t)=\left\langle\Phi\left|e^{i H_{g} t} e^{-i H_{e} t}\right| \Phi\right\rangle,
$$

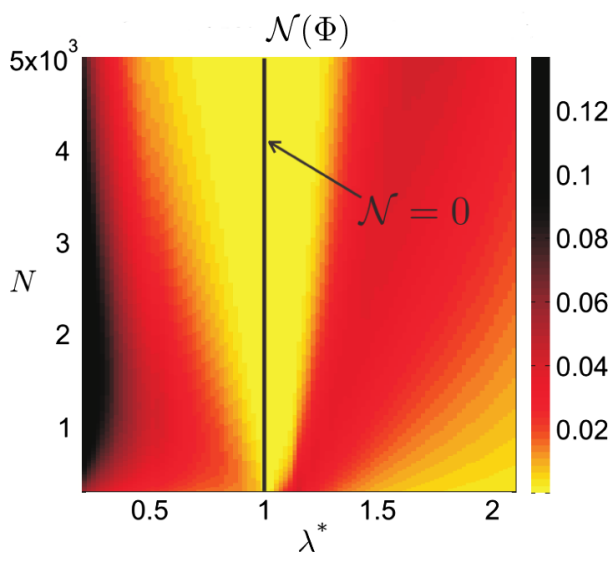

FIG. 6. The non-Markovianity measure $\mathcal{N}(\Phi)$ of Eq. (34) for a qubit coupled to a one-dimensional Ising chain in a transverse field as a function of field strength $\lambda^{*}$ and particle number $N$. The measure vanishes along the black line and is nonzero everywhere else. From Haikka et al., 2012.

where the effective environmental Hamiltonians are $H_{\alpha}=$ $H_{E}+\left\langle\alpha\left|H_{I}\right| \alpha\right\rangle$ with $\alpha=g, e$. We note that according to Eq. (83) the decoherence function is directly linked to the concept of the Loschmidt echo $L(t)$ (Cucchietti et al., 2003) which characterizes how the environment responds to perturbations by the system (Peres, 1984; Cucchietti, Pastawski, and Jalabert, 2004; Gorin et al., 2006; Goussev et al., 2008). In fact, we have $L(t)=|G(t)|^{2}$ and, hence, there is also a direct connection between the Loschmidt echo and the evolution of the trace distance for optimal state pairs, i.e., $D\left(\rho_{S}^{1}(t), \rho_{S}^{2}(t)\right)=\sqrt{L(t)}$.

The system-environment interaction (82) leads to the effective transverse field $\lambda^{*}=\lambda+\delta$ when the system is in the state $|e\rangle$. At the critical point of the spin environment $\left(\lambda^{*}=1\right)$ the spin-spin interaction and the external field are of the same size and the environment is most sensitive to external perturbations. This is reflected in a quite remarkable manner in the dynamics of the system qubit as can be seen from Fig. 6 (Haikka et al., 2012). At the point $\lambda^{*}=1$ the system qubit experiences strong decoherence. As a matter of fact, it turns out that the dynamics of the system qubit always shows nonMarkovian behavior except at the critical point of the environment where the dynamics is Markovian. Therefore, the system qubit acts as a probe for the spin environment and Markovian dynamics represents a reliable indicator of environment criticality. It is important to note that even though, strictly speaking, quantum phase transitions require one to take the thermodynamic limit, these results hold for any number of environmental spins.

\section{IMPACT OF CORRELATIONS AND EXPERIMENTAL REALIZATIONS}

In this section we study the effect of two fundamental kinds of correlations on the non-Markovian behavior of open systems, namely, correlations between the open system and its environment and correlations within a composite environment. We conclude the section by giving an overview of the experimental status of non-Markovian open systems. 


\section{A. Initial system-environment correlations}

\section{Initial correlations and dynamical maps}

Up to this point we have discussed the theory of open quantum systems via the concept of a dynamical map, which in Sec. II.A.1 we wrote down in terms of the initial state of the environment and of the total Hamiltonian. In doing so we assumed that the open system and the environment are independent at the initial time of preparation [see Eqs. (6) and (7)]. However, experimentalists often study fast processes in strongly coupled systems, where this independence is rarely the case, as originally pointed out by Pechukas (1994). Going beyond this approximation brings us to the problem of describing the dynamical properties of open quantum systems without dynamical maps.

The theory of completely positive maps plays a crucial role in many fields of quantum physics and thus a general map description for open systems, even in the presence of initial correlations, would be very useful. Thus, naturally, a lot of effort has been put into trying to extend the formalism along these lines (Alicki, 1995; Stelmachovic and Buzek, 2001; Shabani and Lidar, 2009). In this framework the aim has been to determine under which conditions the system dynamics can be described by means of completely positive maps if initial correlations are present. Unfortunately, no generally acknowledged answer to this question has been found and the topic is still under discussion (Shabani and Lidar, 2009; Rodríguez-Rosario, Modi, and Aspuru-Guzik, 2010; Brodutch et al., 2013).

As useful as a description in terms of completely positive maps would be, one may as well ask the question: How do initial correlations influence the dynamics? Especially, since an experimentalist is often restricted to looking only at a subset of system states, one would want to conclude something about the system based on the dynamics of this subset. It is not possible to construct a map, but perhaps something else could be concluded. In the following we present an approach in which the behavior of the information flow between the system and the environment is studied. We will see that initial correlations modify the information flow and consequently can be witnessed from the dynamical features of the open system.

Let us have a closer look at the dynamics of the information inside the open system $\mathcal{I}_{\text {int }}(t)$, defined in Eq. (30), for a general open system, where initial correlations could be present. Suppose there are two possible preparations giving rise to distinct system-environment initial states $\rho_{S E}^{1}(0)$ and $\rho_{S E}^{2}(0)$. Since the total system and the environment evolve under unitary dynamics, we have

$$
\mathcal{I}_{\text {int }}(t)-\mathcal{I}_{\text {int }}(0)=\mathcal{I}_{\text {ext }}(0)-\mathcal{I}_{\text {ext }}(t) \leq \mathcal{I}_{\text {ext }}(0)
$$

where $\mathcal{I}_{\text {ext }}(t)$ is the information outside the open system defined in Eq. (31). This inequality, as simple as it is, reveals one very important point: The information in the open system $\mathcal{I}_{\text {int }}(t)$ can increase above its initial value $\mathcal{I}_{\text {int }}(0)$ only if there is some information initially outside the system, i.e., $\mathcal{I}_{\text {ext }}(0)>0$. The contraction property (28) for completely positive maps is a special case of the inequality (84) which occurs when the system and the environment are initially uncorrelated and the environmental state is not influenced by the system state preparation, i.e., $\rho_{S E}^{1,2}=\rho_{S}^{1,2} \otimes \rho_{E}$. This leads to $\mathcal{I}_{\text {ext }}(0)=0$ and, thus, Eq. (84) reduces to Eq. (29).

\section{Local detection of initial correlations}

We found that initial information outside the open system can lead to an increase of the trace distance. But how can this be used to develop experimental methods to detect correlations in some unknown initial state $\rho_{S E}^{1}$ ? To this end, let us combine Eq. (84) and the inequality (33) for the initial time in order to reveal the role of initial correlations more explicitly (Laine, Piilo, and Breuer, 2010b):

$$
\begin{aligned}
\mathcal{I}_{\text {int }}(t)-\mathcal{I}_{\text {int }}(0) \leq & D\left(\rho_{S E}^{1}(0), \rho_{S}^{1}(0) \otimes \rho_{E}^{1}(0)\right) \\
& +D\left(\rho_{S E}^{2}(0), \rho_{S}^{2}(0) \otimes \rho_{E}^{2}(0)\right) \\
& +D\left(\rho_{E}^{1}(0), \rho_{E}^{2}(0)\right) .
\end{aligned}
$$

This inequality shows that an increase of the trace distance of the reduced states implies that there are initial correlations in $\rho_{S E}^{1}(0)$ or $\rho_{S E}^{2}(0)$, or that the initial environmental states are different.

Let us now assume that one can perform a state tomography on the open system at the initial time zero and at some later time $t$, to determine the reduced states $\rho_{S}^{1}(0)$ and $\rho_{S}^{1}(t)$. In order to apply the inequality (85) to detect initial correlations we need a second reference state $\rho_{S}^{2}(0)$, which has the same environmental state, i.e., $\rho_{E}^{2}(0)=\rho_{E}^{1}(0)$. This can be achieved by performing a local quantum operation on $\rho_{S E}^{1}(0)$ to obtain the state

$$
\rho_{S E}^{2}(0)=(\Lambda \otimes I) \rho_{S E}^{1}(0)
$$

The operation $\Lambda$ acts locally on the variables of the open system and may be realized, for instance, by the measurement of an observable of the open system, or by a unitary transformation induced, e.g., through an external control field. Now, if $\rho_{S E}^{1}(0)$ is uncorrelated then also $\rho_{S E}^{2}(0)$ is uncorrelated since it has been obtained through a local operation. Therefore, for an initially

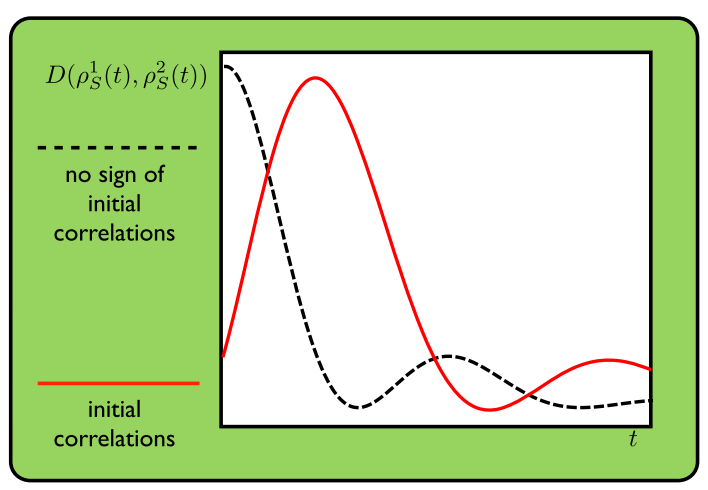

FIG. 7. The behavior of the trace distance with and without initial correlations. If the trace distance between two states, prepared such that $\rho_{S E}^{2}(0)=(\Lambda \otimes I) \rho_{S E}^{1}(0)$, never exceeds its initial value (dashed black line), the dynamics does not witness initial correlations. If, on the other hand, the trace distance for such pair of states increases above the initial value (solid red line), we can conclude the presence of initial correlations. 
uncorrelated state the trace distance cannot increase according to inequality (85). Thus, any such increase represents a witness for correlations in the initial state $\rho_{S E}^{1}(0)$, as is illustrated in Fig. 7. We note that this method for the local detection of initial correlations requires only local control and measurements of the open quantum system, which makes it feasible experimentally and thus attractive for applications. In fact, experimental realizations of the scheme have been reported recently; see Sec. IV.C.2. Furthermore, Smirne et al. (2010) studied how the scheme can be employed for detecting correlations in thermal equilibrium states, while the interplay between nonMarkovianity and the formation of system-environment correlations was studied by Mazzola et al. (2012) and Smirne, Mazzola et al. (2013).

This strategy can even be used in order to locally detect a specific type of quantum correlations, i.e., systemenvironment states with nonzero quantum discord (Modi et al., 2012). This is achieved by taking the local operation $\Lambda$ in Eq. (86) to be a dephasing operation leading to complete decoherence in the eigenbasis of the state $\rho_{S}^{1}(0)$ (Gessner and Breuer, 2011, 2013). The application of this dephasing operation destroys all quantum correlations of $\rho_{S E}^{1}(0)$, while leaving invariant its marginal states $\rho_{S}^{1}(0)$ and $\rho_{E}^{1}(0)$ (see Fig. 8). Thus, one can use the quantity $\mathcal{C}\left(\rho_{S E}^{1}(0)\right)=$ $D\left(\rho_{S E}^{1}(0), \rho_{S E}^{2}(0)\right)$ as a measure for the quantum correlations in the initial state $\rho_{S E}^{1}(0)$ (Luo, 2008). Since the trace distance is invariant under unitary transformations and since the partial trace is a positive map, the corresponding local trace distance $D\left(\rho_{S}^{1}(t), \rho_{S}^{2}(t)\right)$ provides a lower bound of $\mathcal{C}\left(\rho_{S E}^{1}(0)\right)$ for all times $t \geq 0$. Hence, we have

$$
\max _{t \geq 0} D\left(\rho_{S}^{1}(t), \rho_{S}^{2}(t)\right) \leq \mathcal{C}\left(\rho_{S E}^{1}(0)\right)
$$

The left-hand side of this inequality yields a locally accessible lower bound for a measure of the quantum discord. Experimental realizations of the scheme are briefly described in Sec. IV.C.2. Most recent studies suggest that the method could also be applied for detecting a critical point of a quantum phase transition via dynamical monitoring of a single spin alone (Gessner, Ramm, Häffner et al., 2014).

\section{B. Nonlocal memory effects}

We now return to study the case of initially uncorrelated system-environment states for which the usual description in terms of dynamical maps can be used. So far, we have

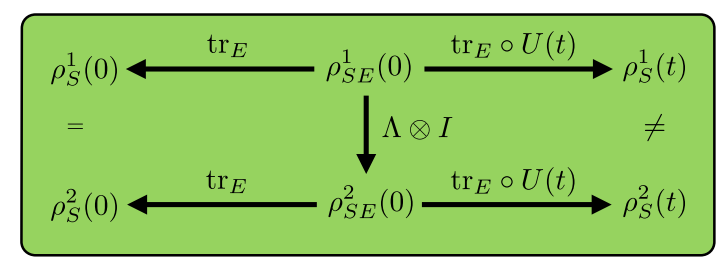

FIG. 8. Scheme for the local detection of system-environment quantum correlations in some state $\rho_{S E}^{1}(0)$, employing a local dephasing operation $\Lambda$ to generate a second reference state $\rho_{S E}^{2}(0)$. concentrated on examples with a single system embedded in an environment. However, many relevant examples in quantum information processing include multipartite systems for which nonlocal properties such as entanglement become important and it is therefore essential to study how nonMarkovianity is influenced by scaling up the number of particles.

The question of additivity of memory effects with respect to particle number was recently raised by Addis et al. (2013, 2014) and Fanchini et al. (2013) and it was found that the different measures for non-Markovianity have very distinct additivity properties. Indeed, the research on multipartite open quantum systems is yet in its infancy and a conclusive analysis remains undone. In the following, we discuss a particular feature of bipartite open systems: the appearance of global memory effects in the absence of local non-Markovian dynamics. This is at variance with the standard situation in which the enlargement of considered degrees of freedom leads from a non-Markovian to a Markovian dynamics (Martinazzo et al., 2011).

We now take a closer look at nonlocal maps, for which memory effects may occur even in the absence of local nonMarkovian effects. Laine et al. $(2012,2013)$ showed that such maps may be generated from a local interaction, when correlations between the environments are present and that they may exhibit dynamics with strong global memory effects although the local dynamics is Markovian. The nonlocal memory effects are studied in two dephasing models: in a generic model of qubits interacting with correlated multimode fields (Wißmann and Breuer, 2013, 2014) and in an experimentally realizable model of downconverted photons traveling through quartz plates, which we discuss later in detail.

Before we discuss any experimental endeavor to detect non-Markovian dynamics, let us discuss in more rigor the generation and possible applications of nonlocal memory effects. Consider a generic scenario, where there are two systems, labeled with indices $i=1,2$, which interact locally with their respective environments. The dynamics of the two systems can be described via the dynamical map

$$
\begin{aligned}
\rho_{S}^{12}(t) & =\Phi_{t}^{12} \rho_{S}^{12}(0) \\
& =\operatorname{tr}_{E}\left[U_{S E}^{12}(t) \rho_{S}^{12}(0) \otimes \rho_{E}^{12}(0) U_{S E}^{12 \dagger}(t)\right]
\end{aligned}
$$

where $U_{S E}^{12}(t)=U_{S E}^{1}(t) \otimes U_{S E}^{2}(t)$ with $U_{S E}^{i}(t)$ describing the local interaction between the system $i$ and its environment. If the initial environment state $\rho_{E}^{12}(0)$ factorizes, i.e., $\rho_{E}^{12}(0)=\rho_{E}^{1}(0) \otimes \rho_{E}^{2}(0)$, also the map $\Phi_{t}^{12}$ factorizes. Thus, the dynamics of the two systems is given by a local map $\Phi_{t}^{12}=\Phi_{t}^{1} \otimes \Phi_{t}^{2}$. On the other hand, if $\rho_{E}^{12}(0)$ exhibits correlations, the map $\Phi_{t}^{12}$ cannot, in general, be factorized. Consequently, the environmental correlations may give rise to a nonlocal process even though the interaction Hamiltonian is purely local (see Fig. 9).

For a local dynamical process, all the dynamical properties of the subsystems are inherited by the global system, but naturally for a nonlocal process the global dynamics can display characteristics absent in the dynamics of the local constituents. Especially, for a nonlocal process, even if the subsystems undergo a Markovian evolution, the global 


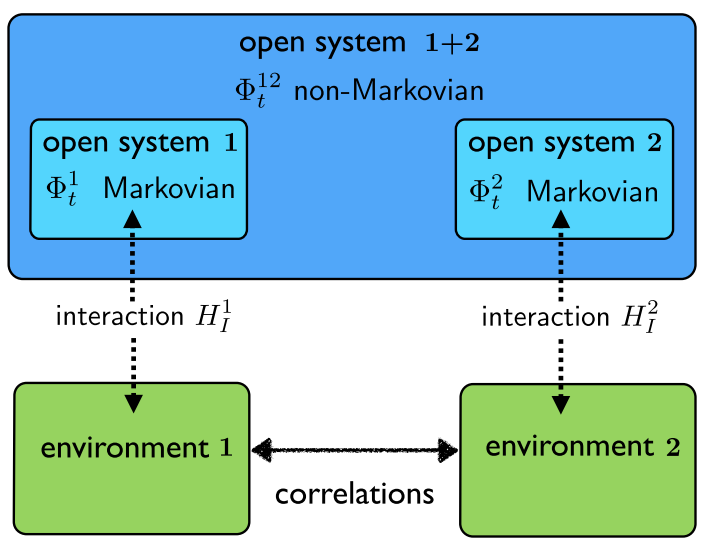

FIG. 9. A system with nonlocal memory effects. The open systems 1 and 2 locally interact with their respective environments. Initial correlations between the local environments cause the occurrence of nonlocal memory effects.

dynamics can nevertheless be highly non-Markovian as was shown for the dephasing models by Laine et al. (2012, 2013).

Thus, a system can globally recover its earlier lost quantum properties although the constituent parts are undergoing decoherence and this way initial environmental correlations can diminish the otherwise destructive effects of decoherence. This feature has been deployed in noisy quantum information protocols, such as teleportation (Laine, Breuer, and Piilo, 2014) and entanglement distribution (Xiang et al., 2014), suggesting that non-Markovianity could be a resource for quantum information.

\section{Experiments on non-Markovianity and correlations}

Up to this point we have not yet discussed any experimental aspects of the detection of memory effects. In the framework of open quantum systems the environment is in general composed of many degrees of freedom and is therefore difficult to access or control. To perform experiments on systems where the environment-induced dynamical features can be controlled is thus challenging. However, in the past years clever schemes for modifying the environment have been developed allowing the establishment of robust designs for noise engineering. In this section we briefly review some of the experimental platforms where a high level of control over the environment degrees of freedom has been accomplished and non-Markovian dynamics observed and quantified.

\section{Control and quantification of memory effects in photonic systems}

Quantum optical experiments have for many decades been the bedrock for testing fundamental paradigms of quantum mechanics. The appeal for using photonic systems arises from the extremely high level of control allowing, for example, controlled interactions between different degrees of freedom, preparation of arbitrary polarization states, and a full state tomography. Needless to say, photons thus offer an attractive experimental platform also for studying non-Markovian effects.
Liu et al. (2011) introduced an all-optical experiment which allows through careful manipulation of the initial environmental states to drive the open system dynamics from the Markovian to the non-Markovian regime, to control the information flow between the system and the environment, and to determine the degree of non-Markovianity. In this experiment the photon polarization degree of freedom (with basis states $|H\rangle$ and $|V\rangle$ for horizontal and vertical polarization, respectively) plays the role of the open system. The environment is represented by the frequency degree of freedom of the photon (with basis $\{|\omega\rangle\}_{\omega \geq 0}$ ), which is coupled to the system via an interaction induced by a birefringent material (quartz plate). The interaction between the polarization and frequency degrees of freedom in the quartz plate of thickness $L$ is described by the unitary operator $U(t)|\lambda\rangle \otimes|\omega\rangle=e^{i n_{\lambda} \omega t}|\lambda\rangle \otimes|\omega\rangle$, where $n_{\lambda}$ is the refraction index for a photon with polarization $\lambda=H, V$, and $t=L / c$ is the interaction time with the speed of light $c$. The photon is initially prepared in the state $|\psi\rangle \otimes|\chi\rangle$, with $|\psi\rangle=\alpha|H\rangle+$ $\beta|V\rangle$ and $|\chi\rangle=\int d \omega f(\omega)|\omega\rangle$, where $f(\omega)$ gives the amplitude for the photon to be in a mode with frequency $\omega$. The quartz plate leads to a pure decoherence dynamics of superpositions of polarization states (see Sec. III.A.1) described by the complex decoherence function $G(t)=\int d \omega|f(\omega)|^{2} e^{i \omega \Delta n t}$, where $\Delta n=n_{V}-n_{H}$. An optimal pair of states maximizing the non-Markovianity measure (34) for this map is given by $\left|\psi_{1,2}\right\rangle=(1 / \sqrt{2})(|H\rangle \pm|V\rangle)$ and the corresponding trace distance is $D\left(\rho_{S}^{1}(t), \rho_{S}^{2}(t)\right)=|G(t)|$. Clearly, controlling the frequency spectrum $|f(\omega)|^{2}$ changes the dynamical features of the map.

The modification of the photon frequency spectrum is realized by means of a Fabry-Pérot cavity mounted on a rotator which can be tilted in the horizontal plane. The shape of the frequency spectrum is changed by changing the tilting angle $\theta$ of the Fabry-Pérot cavity, as can be seen in Fig. 10(a). Further, the frequency spectrum changes the dephasing dynamics such that the open system exhibits a reversed flow of information and thus allows one to tune the dynamics from a Markovian to a non-Markovian regime [see Fig. 10(b)]. In the experiment, full state tomography can further be performed thus allowing a rigorous quantification of the memory effects.

A series of other experimental studies on non-Markovian dynamics in photonic systems were performed recently. Tang et al. (2012) reported a measurement of the non-Markovianity of a process with tunable system-environment interaction, Cialdi et al. (2011) observed controllable entanglement oscillations in an effective non-Markovian channel, and Chiuri et al. (2012) and Jin et al. (2015) presented simulation platforms for a wide class of non-Markovian channels. Moreover, a non-Markovian collision model proposed by Bernardes et al. (2014) was realized quite recently in a photonic experiment (Bernardes et al., 2015).

\section{Experiments on the local detection of correlations}

A number of experiments have been carried out in order to demonstrate the local scheme for the detection of correlations between an open system and its environment described in Sec. IV.A.2. Photonic realizations of this scheme have been 


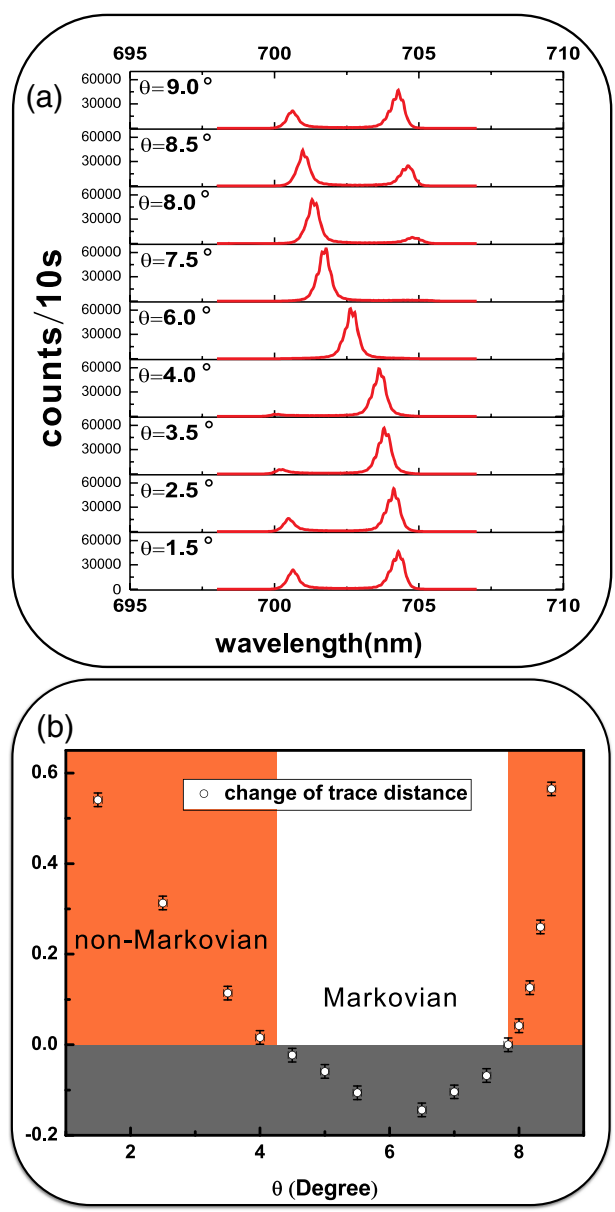

FIG. 10. Non-Markovian dynamics arising from an engineered frequency spectrum. (a) The frequency spectrum of the initial state for various values of the tilting angle $\theta$ of the cavity. (b) The change of the trace distance as a function of the tilting angle $\theta$. The transition from the non-Markovian to the Markovian regime occurs at $\theta \approx 4.1^{\circ}$, and from the Markovian to the nonMarkovian regime at $\theta \approx 8.0^{\circ}$, corresponding to the occurrence of a double peak structure in the frequency spectrum (a). From Liu et al., 2011.

reported by Li et al. (2011) and Smirne et al. (2011), where the presence of initial correlations between the polarization and the spatial degrees of freedom of photons is shown by the observation of an increase of the local trace distance between a pair of initial states.

As explained in Sec. IV.A.2, it is also possible to locally reveal the presence of quantum correlations represented by system-environment states with nonzero discord if the local operation $\Lambda$ in Eq. (86) is taken to induce complete decoherence in the eigenbasis of the open system state. The first photonic realizations of this strategy based on Eq. (87) were described by Tang et al. (2015). Moreover, Cialdi et al. (2014) extended the method to enable the discrimination between quantum and classical correlations and applied this extension to a photonic experimental realization.

All experiments mentioned so far employ photonic degrees of freedom to demonstrate non-Markovianity and systemenvironment correlations. The first experiment showing these phenomena for matter degrees of freedom has been described by Gessner, Ramm, Pruttivarasin et al. (2014). In this experiment nonclassical correlations between the internal electronic degrees of freedom and the external motional degrees of freedom of a trapped ion have been observed and quantified by use of Eq. (87). Important features of the experiment are that the lower bounds obtained from the experimental data are remarkably close to the true quantum correlations present in the initial state and that it also allows the study of the temperature dependence of the effect.

\section{Non-Markovian quantum probes detecting nonlocal correlations in composite environments}

In Sec. IV.B we demonstrated the fact that initial correlations between local parts of the environment can lead to nonlocal memory effects. Liu et al. (2013) realized such nonlocal memory effects in a photonic system, where manipulating the correlations of the photonic environments led to non-Markovian dynamics of the open system. The experimental scheme provided a controllable diagnostic tool for the quantification of these correlations by repeated tomographic measurements of the polarization.

Let us take a closer look at the system under study in the experiment. A general pure initial polarization state of a photon pair can be written as $\left|\psi_{12}\right\rangle=a|H H\rangle+b|H V\rangle+$ $c|V H\rangle+d|V V\rangle$ and all initial states of the polarization plus the frequency degrees of freedom are product states

$$
|\Psi(0)\rangle=\left|\psi_{12}\right\rangle \otimes \int d \omega_{1} d \omega_{2} g\left(\omega_{1}, \omega_{2}\right)\left|\omega_{1}, \omega_{2}\right\rangle,
$$

where $g\left(\omega_{1}, \omega_{2}\right)$ is the probability amplitude for photon 1 to have frequency $\omega_{1}$ and for photon 2 to have frequency $\omega_{2}$, with the corresponding joint probability distribution $P\left(\omega_{1}, \omega_{2}\right)=\left|g\left(\omega_{1}, \omega_{2}\right)\right|^{2}$. If the photons pass through quartz plates, the dynamics can be described by a general two-qubit dephasing map, where the different decoherence functions can be expressed in terms of Fourier transforms of the joint probability distribution $P\left(\omega_{1}, \omega_{2}\right)$. For a Gaussian joint frequency distribution with identical single frequency variances $C$ and correlation coefficient $K$, the time evolution of the trace distance corresponding to the Bell-state pair $\left|\psi_{12}^{ \pm}\right\rangle=$ $(1 / \sqrt{2})(|H H\rangle \pm|V V\rangle)$ is found to be

$$
D(t)=\exp \left[-\frac{1}{2} \Delta n^{2} C\left(t_{1}^{2}+t_{2}^{2}-2|K| t_{1} t_{2}\right)\right]
$$

where $t_{i}$ denotes the time photon $i$ has interacted with its quartz plate up to the actual observation time $t$. For uncorrelated photon frequencies we have $K=0$ and the trace distance decreases monotonically, corresponding to Markovian dynamics. However, as soon as the frequencies are anticorrelated, $K<0$, the trace distance is nonmonotonic which signifies quantum memory effects and non-Markovian behavior. On the other hand, the local frequency distributions are Gaussian and thus for the single photons the trace distance monotonically decreases. Therefore, we concluded that the system is locally Markovian but globally displays nonlocal memory effects.

In the experiment two quartz plates act consecutively for the photons, and the magnitude of the initial anticorrelations 
between the local reservoirs is tuned. After the photon exits the quartz plates, full two-photon polarization state tomography is performed and by changing the quartz plate thicknesses, the trace distance dynamics is recovered. From the dynamics it is evident that initial environmental correlations influence the quantum non-Markovianity.

A further important aspect of the experimental scheme is that it enables one to determine the frequency correlation coefficient $K$ of the photon pairs from measurements performed on the polarization degree of freedom. By performing tomography on a small system we can obtain information on frequency correlations, difficult to measure directly. Thus, the open system (polarization degrees of freedom) can serve as a quantum probe which allows us to gain nontrivial information on the correlations in the environment (frequency degrees of freedom).

\section{SUMMARY AND OUTLOOK}

In this Colloquium we presented recent advances in the definition and characterization of non-Markovian dynamics of open quantum systems. While in the classical case the very definition of Markovian stochastic process can be explicitly given in terms of constraints on the conditional probabilities of the process, in the quantum realm the peculiar role of measurements prevents a direct formulation along the same path and new approaches are called for in order to describe memory effects. We therefore introduced a notion of nonMarkovianity of a quantum dynamical map based on the behavior in time of the distinguishability of different system states, as quantified by their trace distance, which provides an intrinsic characterization of the dynamics. This approach allows one to connect non-Markovianity with the flow of information from the environment back to the system and naturally leads to the introduction of quantum information concepts. It is further shown to be connected to other approaches recently presented in the literature building on divisibility properties of the quantum dynamical map. In particular, a generalization of the trace distance criterion allows one to identify Markovian time evolutions with quantum evolutions which are $P$ divisible, thus leading to a clear-cut connection between memory effects in the classical and quantum regimes. As a crucial feature the trace distance approach to non-Markovianity can be experimentally tested and allows for the study of system-environment correlations as well as nonlocal memory effects emerging from correlations within the environment.

We have illustrated the basic features of quantum nonMarkovianity by analyzing in detail simple paradigmatic model systems and by further discussing more complex models which exhibit important connections between nonMarkovianity of the open system dynamics and fundamental properties of the environment. Indeed, it is possible to obtain information on a complex quantum system (environment) via the study of the dynamics of a small quantum probe (open system) coupled to it. The time development of the trace distance between states of the quantum probe can be shown to yield a witness for characteristic properties of the complex environment, for example, the presence of a quantum phase transition, and leads to methods for the detection and quantification of correlations within the environment. Moreover, the observation of non-Markovian dynamics provides a tool for the local detection of system-environment correlations and even for the distinction between the classical and quantum nature of such correlations.

Experimentally, it is in general very difficult to control in detail the environmental degrees of freedom and therefore experiments on non-Markovian quantum systems are still in a state of infancy. Still, some sophisticated schemes for modifying the environment have been developed, thus leading to the realization of first proof-of-principle experiments in which to test the study of non-Markovianity, its connection with crucial features of the environment, as well as the capability to unveil system-environment correlations by means of local observations on the system.

In recent years non-Markovian quantum systems have been enjoying much attention due to both fundamental reasons and foreseeable applications as shown by the rapid growth in the related literature. Indeed, the potential relevance of memory effects in the field of complex quantum systems and quantum information has led to an intense study, and we pointed to some highlights in this novel, so far fairly unexplored field of nonMarkovianity. However, there are numerous open questions yet to be studied. The latter include fundamental questions such as the mathematical structure of the space of non-Markovian quantum dynamical maps, the role of complexity in the emergence of memory effects or the relevance of nonMarkovianity in the study of the border between classical and quantum aspects of nature, as well as more applied issues such as the identification of the environmental features or system-environment correlations which can indeed be detected by means of local observations on the system. A further fruitful line of research could be to examine the role of quantum nonMarkovianity and optimal state pairs in quantum control and dynamical decoupling schemes [see, e.g., Viola, Knill, and Lloyd (1999) and Wiseman and Milburn (2010)].

The theoretical and experimental investigations of nonMarkovian quantum systems outlined in this Colloquium pave the way for new lines of research both by shedding light on fundamental questions of open quantum systems and by suggesting novel applications in quantum information and probing of complex systems.

\section{ACKNOWLEDGMENTS}

We acknowledge support from the European Union (EU) through the Collaborative Project QuProCS (Grant Agreement No. 641277). J. P. and B. V. acknowledge support from the COST MP1006 Fundamental Problems in Quantum Physics, E. M. L. from the Academy of Finland through its Centres of Excellence Programme (2012-2017) under Project No. 251748, J.P. from the Jenny and Antti Wihuri Foundation and the Magnus Ehrnrooth Foundation, and B. V. from the Unimi Transition Grant-Horizon 2020.

\section{REFERENCES}

Accardi, L., A. Frigerio, and J. T. Lewis, 1982, Publ. RIMS Kyoto Univ. 18, 97. 
Addis, C., B. Bylicka, D. Chruściński, and S. Maniscalco, 2014, Phys. Rev. A 90, 052103.

Addis, C., P. Haikka, S. McEndoo, C. Macchiavello, and S. Maniscalco, 2013, Phys. Rev. A 87, 052109.

Alicki, R., 1995, Phys. Rev. Lett. 75, 3020.

Alicki, R., and K. Lendi, 1987, Quantum Dynamical Semigroups and Applications, Lecture Notes in Physics, Vol. 286 (Springer, Berlin).

Alicki, R., D. A. Lidar, and P. Zanardi, 2006, Phys. Rev. A 73, 052311.

Apollaro, T. J. G., C. Di Franco, F. Plastina, and M. Paternostro, 2011, Phys. Rev. A 83, 032103.

Apollaro, T. J. G., S. Lorenzo, C. Di Franco, F. Plastina, and M. Paternostro, 2014, Phys. Rev. A 90, 012310.

Bernardes, N. K., A. R. R. Carvalho, C. H. Monken, and M. F. Santos, 2014, Phys. Rev. A 90, 032111.

Bernardes, N. K., A. Cuevas, A. Orieux, C. H. Monken, P. Mataloni, F. Sciarrino, and M. F. Santos, 2015, Sci. Rep. 5, 17520.

Borrelli, M., P. Haikka, G. deChiara, and S. Maniscalco, 2013, Phys. Rev. A 88, 010101(R).

Breuer, H.-P., 2012, J. Phys. B 45, 154001.

Breuer, H.-P., B. Kappler, and F. Petruccione, 1999, Phys. Rev. A 59, 1633.

Breuer, H.-P., E.-M. Laine, and J. Piilo, 2009, Phys. Rev. Lett. 103, 210401.

Breuer, H.-P., and F. Petruccione, 2002, The Theory of Open Quantum Systems (Oxford University Press, Oxford).

Brodutch, A., A. Datta, K. Modi, A. Rivas, and C. A. RodríguezRosario, 2013, Phys. Rev. A 87, 042301.

Buscemi, F., and N. Datta, 2014 arXiv:1408.7062.

Bylicka, B., D. Chruściński, and S. Maniscalco, 2014, Sci. Rep. 4, 5720.

Chancellor, N., C. Petri, and S. Haas, 2013, Phys. Rev. B 87, 184302.

Chaturvedi, S., and F. Shibata, 1979, Z. Phys. B 35, 297.

Chin, A. W., S. F. Huelga, and M. B. Plenio, 2012, Phys. Rev. Lett. 109, 233601.

Chiuri, A., C. Greganti, L. Mazzola, M. Paternostro, and P. Mataloni, 2012, Sci. Rep. 2, 968.

Chruściński, D., and A. Kossakowski, 2012, J. Phys. B 45, 154002.

Chruściński, D., A. Kossakowski, and A. Rivas, 2011, Phys. Rev. A 83, 052128 .

Chruściński, D., and S. Maniscalco, 2014, Phys. Rev. Lett. 112, 120404.

Chruściński, D., and F. A. Wudarski, 2013, Phys. Lett. A 377, 1425.

Cialdi, S., D. Brivio, E. Tesio, and M. G. A. Paris, 2011, Phys. Rev. A 83, 042308.

Cialdi, S., A. Smirne, M. G. A. Paris, S. Olivares, and B. Vacchini, 2014, Phys. Rev. A 90, 050301.

Clos, G., and H.-P. Breuer, 2012, Phys. Rev. A 86, 012115.

Cucchietti, F. M., D. A. R. Dalvit, J. P. Paz, and W. H. Zurek, 2003, Phys. Rev. Lett. 91, 210403.

Cucchietti, F. M., H. M. Pastawski, and R. A. Jalabert, 2004, Phys. Rev. B 70, 035311.

Dajka, J., J. Łuczka, and P. Hänggi, 2011, Phys. Rev. A 84, 032120.

Dalfovo, F., S. Giorgini, L. P. Pitaevskii, and S. Stringari, 1999, Rev. Mod. Phys. 71, 463.

Davies, E. B., 1976, Quantum Theory of Open Systems (Academic Press, London).

Dhar, H. S., M. N. Bera, and G. Adesso, 2015, Phys. Rev. A 91, 032115.

Fanchini, F. F., G. Karpat, B. Cakmak, L. K. Castelano, G. H. Aguilar, O. J. Farias, S. P. Walborn, P. H. S. Ribeiro, and M. C. de Oliveira, 2014, Phys. Rev. Lett. 112, 210402.
Fanchini, F. F., G. Karpat, L. K. Castelano, and D. Z. Rossatto, 2013, Phys. Rev. A 88, 012105.

Fuchs, C. A., and J. van de Graaf, 1999, IEEE Trans. Inf. Theory $\mathbf{4 5 ,}$ 1216.

Gardiner, C. W., 1985, Handbook of Stochastic Methods for Physics, Chemistry and the Natural Sciences (Springer, Berlin).

Gessner, M., and H.-P. Breuer, 2011, Phys. Rev. Lett. 107, 180402. Gessner, M., and H.-P. Breuer, 2013, Phys. Rev. A 87, 042107.

Gessner, M., M. Ramm, H. Häffner, A. Buchleitner, and H.-P. Breuer, 2014, Europhys. Lett. 107, 40005.

Gessner, M., M. Ramm, T. Pruttivarasin, A. Buchleitner, H.-P. Breuer, and H. Häffner, 2014, Nat. Phys. 10, 105.

Gorin, T., T. Prosen, T. H. Seligman, and M. Žnidarič, 2006, Phys. Rep. 435, 33.

Gorini, V., A. Kossakowski, and E. C. G. Sudarshan, 1976, J. Math. Phys. (N.Y.) 17, 821.

Goussev, A., D. Waltner, K. Richter, and R. A. Jalabert, 2008, New J. Phys. 10, 093010.

Gröblacher, S., A. Trubarov, N. Prigge, G. D. Cole, M. Aspelmeyer, and J. Eisert, 2015, Nat. Commun. 6, 7606.

Guarnieri, G., A. Smirne, and B. Vacchini, 2014, Phys. Rev. A 90, 022110 .

Haikka, P., J. Goold, S. McEndoo, F. Plastina, and S. Maniscalco, 2012, Phys. Rev. A 85, 060101.

Haikka, P., T. Johnson, and S. Maniscalco, 2013, Phys. Rev. A 87, 010103(R).

Haikka, P., S. McEndoo, G. De Chiara, G. M. Palma, and S. Maniscalco, 2011, Phys. Rev. A 84, 031602.

Hall, M. J. W., J. D. Cresser, L. Li, and E. Andersson, 2014, Phys. Rev. A 89, 042120.

Haseli, S., G. Karpat, S. Salimi, A. S. Khorashad, F. F. Fanchini, B. Cakmak, G. H. Aguilar, S. P. Walborn, and P. H. S. Ribeiro, 2014, Phys. Rev. A 90, 052118.

Heinosaari, T., and M. Ziman, 2011, The Mathematical Language of Quantum Theory (Cambridge University Press, Cambridge, England).

Helstrom, C. W., 1967, Inf. Control 10, 254.

Helstrom, C. W., 1976, Quantum Detection and Estimation Theory (Academic Press, New York).

Hou, S. C., X. X. Yi, S. X. Yu, and C. H. Oh, 2011, Phys. Rev. A 83, 062115.

Huelga, S. F., A. Rivas, and M. B. Plenio, 2012, Phys. Rev. Lett. 108, 160402.

Jin, J., V. Giovannetti, R. Fazio, F. Sciarrino, P. Mataloni, A. Crespi, and R. Osellame, 2015, Phys. Rev. A 91, 012122.

Kossakowski, A., 1972a, Bull. Acad. Pol. Sci., Sér. Sci. Math. Astron. Phys. 20, 1021.

Kossakowski, A., 1972b, Rep. Math. Phys. 3, 247.

Kraus, K., 1983, States, Effects, and Operations, Lecture Notes in Physics, Vol. 190 (Springer, Berlin).

Laine, E.-M., H.-P. Breuer, and J. Piilo, 2014, Sci. Rep. 4, 4620.

Laine, E.-M., H.-P. Breuer, J. Piilo, C.-F. Li, and G.-C. Guo, 2012, Phys. Rev. Lett. 108, 210402.

Laine, E.-M., H.-P. Breuer, J. Piilo, C.-F. Li, and G.-C. Guo, 2013, Phys. Rev. Lett. 111, 229901.

Laine, E.-M., J. Piilo, and H.-P. Breuer, 2010a, Phys. Rev. A 81, 062115 .

Laine, E.-M., J. Piilo, and H.-P. Breuer, 2010b, Europhys. Lett. 92, 60010.

Leggett, A. J., S. Chakravarty, A. T. Dorsey, M. P. A. Fisher, A. Garg, and W. Zwerger, 1987, Rev. Mod. Phys. 59, 1.

Li, C.-F., J.-S. Tang, Y.-L. Li, and G.-C. Guo, 2011, Phys. Rev. A 83, 064102. 
Lindblad, G., 1976, Commun. Math. Phys. 48, 119.

Lindblad, G., 1979, Commun. Math. Phys. 65, 281.

Liu, B.-H., D.-Y. Cao, Y.-F. Huang, C.-F. Li, G.-C. Guo, E.-M. Laine, H.-P. Breuer, and J. Piilo, 2013, Sci. Rep. 3, 1781.

Liu, B.-H., L. Li, Y.-F. Huang, C.-F. Li, G.-C. Guo, E.-M. Laine, H.-P. Breuer, and J. Piilo, 2011, Nat. Phys. 7, 931.

Liu, B.-H., S. Wißmann, X.-M. Hu, C. Zhang, Y.-F. Huang, C.-F. Li, G.-C. Guo, A. Karlsson, J. Piilo, and H.-P. Breuer, 2014, Sci. Rep. 4, 6327.

Liu, J., X.-M. Lu, and X. Wang, 2013, Phys. Rev. A 87, 042103.

Lorenzo, S., F. Plastina, and M. Paternostro, 2013, Phys. Rev. A 88, 020102.

Lu, X.-M., X. Wang, and C. P. Sun, 2010, Phys. Rev. A 82, 042103. Luo, S., 2008, Phys. Rev. A 77, 022301.

Luo, S., S. Fu, and H. Song, 2012, Phys. Rev. A 86, 044101.

Martinazzo, R., B. Vacchini, K. H. Hughes, and I. Burghardt, 2011, J. Chem. Phys. 134, 011101.

Mazzola, L., E.-M. Laine, H.-P. Breuer, S. Maniscalco, and J. Piilo, 2010, Phys. Rev. A 81, 062120.

Mazzola, L., C. A. Rodríguez-Rosario, K. Modi, and M. Paternostro, 2012, Phys. Rev. A 86, 010102.

Modi, K., A. Brodutch, H. Cable, T. Paterek, and V. Vedral, 2012, Rev. Mod. Phys. 84, 1655.

Nakajima, S., 1958, Prog. Theor. Phys. 20, 948.

Nielsen, M. A., and I. L. Chuang, 2000, Quantum Computation and Quantum Information (Cambridge University Press, Cambridge, England).

Pechukas, P., 1994, Phys. Rev. Lett. 73, 1060.

Peres, A., 1984, Phys. Rev. A 30, 1610.

Pethick, C. J., and H. Smith, 2008, Bose-Einstein Condensation in Dilute Gases (Cambridge University Press, Cambridge, England), 2nd ed.

Pitaevskii, L., and S. Stringari, 2003, Bose-Einstein Condensation (Oxford University Press, Oxford).

Quan, H. T., Z. Song, X. F. Liu, P. Zanardi, and C. P. Sun, 2006, Phys. Rev. Lett. 96, 140604.

Rebentrost, P., and A. Aspuru-Guzik, 2011, J. Chem. Phys. 134, 101103.

Rivas, A., S. F. Huelga, and M. B. Plenio, 2010, Phys. Rev. Lett. 105, 050403.

Rivas, A., S. F. Huelga, and M. B. Plenio, 2014, Rep. Prog. Phys. 77, 094001.

Rodríguez-Rosario, C. A., K. Modi, and A. Aspuru-Guzik, 2010, Phys. Rev. A 81, 012313.

Ruskai, M. B., 1994, Rev. Math. Phys. 06, 1147.
Sachdev, S., 2011, Quantum Phase Transitions (Cambridge University Press, Cambridge, England).

Shabani, A., and D. A. Lidar, 2009, Phys. Rev. Lett. 102, 100402.

Shibata, F., Y. Takahashi, and N. Hashitsume, 1977, J. Stat. Phys. 17, 171.

Smirne, A., H.-P. Breuer, J. Piilo, and B. Vacchini, 2010, Phys. Rev. A 82, 062114.

Smirne, A., D. Brivio, S. Cialdi, B. Vacchini, and M. G. A. Paris, 2011, Phys. Rev. A 84, 032112.

Smirne, A., S. Cialdi, G. Anelli, M. G. A. Paris, and B. Vacchini, 2013, Phys. Rev. A 88, 012108.

Smirne, A., L. Mazzola, M. Paternostro, and B. Vacchini, 2013, Phys. Rev. A 87, 052129.

Stelmachovic, P., and V. Buzek, 2001, Phys. Rev. A 64, 062106.

Tang, J.-S., C.-F. Li, Y.-L. Li, X.-B. Zou, G.-C. Guo, H.-P. Breuer, E.-M. Laine, and J. Piilo, 2012, Europhys. Lett. 97, 10002.

Tang, J.-S., et al., 2015, Optica 2, 1014.

Vacchini, B., 2012, J. Phys. B 45, 154007.

Vacchini, B., A. Smirne, E.-M. Laine, J. Piilo, and H.-P. Breuer, 2011, New J. Phys. 13, 093004.

van Kampen, N.G., 1992, Stochastic Processes in Physics and Chemistry (North-Holland, Amsterdam).

Vasile, R., S. Maniscalco, M. G. A. Paris, H.-P. Breuer, and J. Piilo, 2011, Phys. Rev. A 84, 052118.

Vasile, R., S. Olivares, M. Paris, and S. Maniscalco, 2011, Phys. Rev. A 83, 042321.

Viola, L., E. Knill, and S. Lloyd, 1999, Phys. Rev. Lett. 82, 2417.

Wiseman, H. M., and G. J. Milburn, 2010, Quantum Measurement and Control (Cambridge University Press, Cambridge, England).

Wißmann, S., and H.-P. Breuer, 2013, arXiv:1310.7722.

Wißmann, S., and H.-P. Breuer, 2014, Phys. Rev. A 90, 032117.

Wißmann, S., H.-P. Breuer, and B. Vacchini, 2015, Phys. Rev. A 92 , 042108 .

Wißmann, S., A. Karlsson, E.-M. Laine, J. Piilo, and H.-P. Breuer, 2012, Phys. Rev. A 86, 062108.

Wißmann, S., B. Leggio, and H.-P. Breuer, 2013, Phys. Rev. A 88, 022108 .

Wolf, M. M., J. Eisert, T. S. Cubitt, and J. I. Cirac, 2008, Phys. Rev. Lett. 101, 150402.

Xiang, G.-Y., Z.-B. Hou, C.-F. Li, G.-C. Guo, H.-P. Breuer, E.-M. Laine, and J. Piilo, 2014, Europhys. Lett. 107, 54006.

Xu, Z. Y., W. L. Yang, and M. Feng, 2010, Phys. Rev. A 81, 044105.

Znidaric, M., C. Pineda, and I. García-Mata, 2011, Phys. Rev. Lett. 107, 080404.

Zwanzig, R., 1960, J. Chem. Phys. 33, 1338. 